\title{
Comparison between Gradient-free and Adjoint Based Aerodynamic Optimization of a Flying Wing Transport Aircraft in the Preliminary Design
}

\author{
Markus Widhalm, ${ }^{*}$ Arno Ronzheimer, ${ }^{\dagger}$ \\ Martin Hepperle $\ddagger$ \\ Insitut of Aerodynamics and Flow Technology, Lilienthalplatz 7, 38108 Braunschweig, Germany
}

\begin{abstract}
This paper describes investigations of planform and shape optimizations for a flying wing transport aircraft with an Euler continuous adjoint method. For a prescribed lift the cost function will be the drag, which has to be minimized and implies a maximization of L/D. The results presented for the gradient based method using an adjoint solver are compared to an optimization performed with a gradient free approach. The different workflows and procedures will demonstrate the advantages and disadvantages of each particular optimization approach.

The optimization procedure uses a freeform deformation technique for the design parameterization of the shape as well as for the surface mesh deformation required to determine the gradients. This combination of both tasks into one tool is advantageous because performing finite differences for evaluating the mesh sensitivities can be carried out with a constant grid topology. An example design case is also shown for demonstration purposes.
\end{abstract}

\section{Nomenclature}

$C_{L} \quad$ lift coefficient

$C_{D}$ drag coefficient

$C_{M Y}$ pitching moment coefficient

$L / D$ Lift-to-drag ratio

$D$ design vector

$F \quad$ flux density tensor

$I$ cost function

$R$ residuals of the flow

$W \quad$ vector of flow variables

$X \quad$ vector of grid coordinates

$\Lambda \quad$ vector of adjoint variables, Lagrangian multiplier

$n \quad$ unit normal surface element in $\mathrm{x}$, $\mathrm{y}$ and $\mathrm{y}$-direction

$v \quad$ velocity vector in $\mathrm{x}, \mathrm{y}$ and $\mathrm{y}$-direction

Subscript

$k \quad$ variable number

\section{Introduction}

Traditional preliminary aerodynamic design methods for aircraft require several changes of the geometry until it satisfies all specifications. Constraints, such as a passenger compartment, trim and stability, have

*Research Scientist, Numerical Methods Branch, Lilienthalplatz 7, 38108 Braunschweig

${ }^{\dagger}$ Research Scientist, Transport Aircraft Branch, Lilienthalplatz 7, 38108 Braunschweig

${ }^{\ddagger}$ Research Scientist, Configuration Design Branch, Lilienthalplatz 7, 38108 Braunschweig 
to be considered. A manual design procedure is a time consuming effort. New approaches in the field of optimization can nowadays reduce the time required for this iterative design process.

Presently, in the preliminary design phase mainly inviscid flow field computations are performed to compensate for the increasing demands concerning accuracy and number of design variables. For the inviscid analyses computational grids are generated quickly and the main effects can be controlled by changing sweep angles, twist and chord length distributions. Viscous effects are often taken into account by simple methods like stripwise boundary layer integration. For the final design steps full viscous computations and windtunnel tests ensure the desired performance of the aircraft. To accelerate the work-flow an optimization with the required constraints is introduced.

In the field of gradient based design, an adjoint method is an efficient and accurate method to evaluate the sensitivities of a cost function. In contrast to many other approaches, Ref. ${ }^{1,13,33}$ the computational cost of the adjoint method does not scale with the number of design variables. Future aerodynamic design applications rely on this feature which is of great interest to refine the optimization process. Two different adjoint approaches can be found in the literature. Either the continuous adjoint, Ref. ${ }^{2,9,14,21}$ by deriving the exact adjoint equation and then linearizing it, or the discrete adjoint, Ref., ${ }^{6,7,19,24}$ by directly deriving it from the discretized flow equations. Both approaches compute the adjoint variables by an additional linear system of equations. Afterwards, together with the mesh sensitivities the final sensitivities, or gradients, are evaluated and transferred to an optimizer.

The presented sensitivity computation relies on the restriction of a constant grid topology. A reliable surface mesh parametrization is essential to keep the effort within limits. For example Hicks-Henne ${ }^{13}$ functions have been used with success in two-dimensional flows, Ref. ${ }^{3,12,17,35}$ For three-dimensional parameterizations we apply the freeform deformation technique, Ref. ${ }^{25-28}$ This technique combines two features in one. The surface parametrization and mesh deformation can be made in a reasonable time.

The comparison of different approaches, such as gradient free and gradient based methods, requires a careful investigation of the design parameters chosen. Only few approaches for pure planform or mixed with shape design parameters can be found in the literature, Ref. ${ }^{16}$ Additionally, it is advisable to use a configuration which is not too complex to point out differences between the individual methods. Otherwise, one may run into problems not related to the optimization procedure. The aircraft investigated here is a blended wing body configuration with a center wing, outboard wing and a transition region, connecting both wings. An unstructured grid is chosen due to its flexibility in creating grids for this particular configuration type. A passenger compartment inside the center wing demonstrates how to take geometrical constraints into account.

\section{Numerical Approach}

The fluid flow affecting the object of interest is simulated with the DLR TAU-Code, Ref. ${ }^{8,10}$ The unsteady TAU-Code solves the compressible, three-dimensional Reynolds-Averaged Navier-Stokes equations using a finite volume formulation. The TAU-Code is based on a hybrid unstructured-grid approach, which makes use of the advantages of semi-structured prismatic grids in the viscous shear layers near walls, and the flexibility in grid generation offered by tetrahedral grids in the surrounding flow volume. The grids used for flow simulations in this paper were created with the hybrid grid generation software Centaur, developed by Centaur Soft. ${ }^{5}$ A dual-grid approach is used in order to make the flow solver independent from the cell types used in the initial grid. The TAU-Code consists of several different modules, including:

- The Preprocessor module, which uses the information from the initial grid to create a dual-grid and secondly coarser grids for multi-grid.

- The Solver module, which performs the flow calculations on the dual-grid.

- The Deformation module, which propagates the deformation of surface grid points to the surrounding volume grid.

- The Postprocessing module, which is used to convert result-files to formats usable by popular visualization tools.

The Euler continuous adjoint method is implemented in the Solver module. The freeform deformation technique, described below, is part of the Deformation module. This approach makes them easy available inside the main TAU workflow. 


\section{II..1. Review of the continuos adjoint approach}

The implementation of the continuous adjoint formulation in the TAU-Code is related to previous works of Ref. ${ }^{2,11,15}$ In this approach the adjoint of the governing equations with respect to a given cost function is derived, before being discretized. The total derivative of a cost function $I$ due to a design parameter alteration of $D$ maybe written as:

$$
\frac{d I}{d D}=\frac{\partial I}{\partial D}+\frac{\partial I}{\partial W} \frac{\partial W}{\partial D} .
$$

In an optimization framework, such a quantity is often referred as sensitivity of $I$. The first term of Eq. (1) expresses the direct effect of the geometry perturbation and the second term contains the effect of the flow alteration caused by the geometry perturbation on the cost function $I$. Solving the above equation can be done by applying finite differences or using the adjoint formulation which allows to calculate the second term without computing the flow solution in the perturbed geometry. Within a flow simulation, the cost function $I$ usually is a functional of the form:

$$
I(D, W(D))=\int_{\Omega} f(D, W(D)) d \Omega .
$$

and in case $f$ has no dependancy from the time and from the time derivatives of $W$ we can write the total derivative of $I$ :

$$
\frac{d I}{d D}=\frac{\partial I}{\partial D}+\int_{\Omega} \frac{\partial f}{\partial W} \frac{\partial W}{\partial D} d \Omega
$$

The adjoint equation follows by adding a term $\Lambda^{T} R$ to Eq. (3) with an arbitrary vector $\Lambda$, a Lagrangian multiplier, and $R$ is the residual from the flow computation and is expressed as:

$$
\begin{aligned}
& R=\int_{\Omega} \nabla \cdot F d \Omega \text { and quasilinear } \quad R=\int_{\Omega} \frac{\partial F}{\partial W} \frac{\partial W}{\partial X} d \Omega=0 . \\
& \text { total derivative of R w.r.t. } \mathrm{D} \quad \frac{d R}{d D}=\frac{\partial R}{\partial D}+\frac{\partial R}{\partial W} \frac{\partial W}{\partial D}
\end{aligned}
$$

With the total derivative of $R$ with respect to the design parameters $D$ the sensitivity of $I$ can be rewritten as:

$$
\begin{aligned}
\frac{d I}{d D} & =\frac{\partial I}{\partial D}+\int_{\Omega} \frac{\partial f}{\partial W} \frac{\partial W}{\partial D} d \Omega+\int_{\Omega} \Lambda^{T}\left(\frac{\partial R}{\partial D}+\frac{\partial R}{\partial W} \frac{\partial W}{\partial D}\right) d \Omega= \\
& =\frac{\partial I}{\partial D}+\int_{\Omega} \Lambda^{T} \frac{\partial R}{\partial D} d \Omega+\int_{\Omega}\left(\frac{\partial f}{\partial W}+\Lambda^{T} \frac{\partial R}{\partial W}\right) \frac{\partial W}{\partial D} d \Omega .
\end{aligned}
$$

The adjoint equation is now

$$
\frac{\partial f}{\partial W}+\Lambda^{T} \frac{\partial R}{\partial W}=0
$$

and we can fulfill the equation by finding the appropriate adjoint variables $\Lambda$, which satisfies the above equation and finally we derive the sensitivity with

$$
\frac{d I}{d D}=\frac{\partial I}{\partial D}+\int_{\Omega} \Lambda^{T} \frac{\partial R}{\partial D} d \Omega
$$

For computing $\mathrm{R}$ we take the three-dimensional compressible Euler equations and the governing equations are given by:

$$
\frac{\partial F}{\partial X}=0
$$

and applying the slip wall boundary condition on the surface $v_{i} n_{i}=0$. Eq. (9) can be represented in a quasilinear form,

$$
\frac{\partial F}{\partial W} \frac{\partial W}{\partial X}=0
$$

where $\partial F / \partial W$ is the flux Jacobian matrice. With the adjoint equation Eq. (7)

$$
\frac{\partial f}{\partial W}+\Lambda^{T} \frac{\partial R}{\partial W}=0 \quad \text { equivalent to }\left(\frac{\partial \Lambda}{\partial X}\right)^{T} \frac{\partial F}{\partial W}=0
$$


we derive the continuous Euler adjoint equation

$$
-\left(\frac{\partial F}{\partial W}\right)^{T} \frac{\partial \Lambda}{\partial X}=0 .
$$

For solving Eq. (12) in a continuous approach, boundary conditions for the Euler wall have to be introduced by expressing the cost functions, e.g. drag or lift, with the dimensional pressure as outlined in Ref. ${ }^{9,14} \Lambda$ has the entries $\psi_{i}$.

$$
n_{x} \psi_{2}+n_{y} \psi_{3}+n_{z} \psi_{4}+d(I)=0 .
$$

and the farfield boundary condition becomes $\Lambda \rightarrow 0$. Choosing the drag minimization as a cost function the final boundary condition can be written as:

$$
n_{x} \psi_{2}+n_{y} \psi_{3}+n_{z} \psi_{4}+\frac{2}{\gamma M_{\infty}^{2} p_{\infty} C_{r e f}}\left(n_{x} \cos \alpha-n_{z} \sin \alpha\right)=0 .
$$

After having derived the adjoint equations the method is completely described. It is obvious by comparing the nonlinear Euler Eq. (10) and linear Euler adjoint Eq. (12) that it can be discretized accordingly to the flow solver by applying a finite volume scheme and either use an explicit time stepping or a semi implicit LUSGS scheme, Ref. ${ }^{6}$

Finally we have to consider evaluating the derivatives of $I$ from Eq. (8) where the first term is the direct effect of the mesh perturbation on the cost function and the second term is the effect of a mesh perturbation induced by a design parameter, while keeping the flow field constant. The total derivative of the remaining cost function $I$, Eq. (8), with respect to $X$ may be written as:

$$
\frac{d I}{d D}=\frac{\partial I}{\partial D}+\frac{\partial I}{\partial X} \frac{\partial X}{\partial D}+\int_{\Omega} \Lambda^{T}\left(\frac{\partial R}{\partial D}+\frac{\partial R}{\partial X} \frac{\partial X}{\partial D}\right) d \Omega .
$$

In our case, the used design parameters deliver no direct derivatives of $I,(\partial I / \partial D)$ and from $R,(\partial R / \partial D)$ and become zero. The following sensitivity equation remains:

$$
\frac{d I}{d D}=\frac{\partial I}{\partial X} \frac{\partial X}{\partial D}+\int_{\Omega} \Lambda^{T}\left(\frac{\partial R}{\partial X} \frac{\partial X}{\partial D}\right) d \Omega .
$$

We can evaluate the metric term variation by simply using finite differences, which is in case of $R$ computed like:

$$
\frac{\partial R}{\partial X} \frac{\partial X}{\partial D_{k}} \approx \frac{\left(R\left(W, X\left(D+\Delta D_{k}\right)\right)-R(W, X)\right)}{\Delta D_{k}}=\frac{\Delta R}{\Delta D_{k}} .
$$

After obtaining $\Lambda$, the sensitivities can be evaluated with a single point deformation and yields for each design variable $D_{k}$ to a variation of the cost function due to the perturbated geometry and we get a scalar difference for the direct variation and a matrix-vector product for the dependency of the Residual,

$$
\frac{d I}{d D_{k}} \approx \frac{\Delta I}{\Delta D_{k}}+\sum_{i}^{\text {npoints }} \Lambda^{T} \frac{\Delta R}{\Delta D_{k}} .
$$

\section{II..2. Surface parametrization and deformation}

The computation of mesh sensitivities is dependent on the deformation of the volume grid, which furthermore depends on the surface grid deformation. The surface grid deformation is performed via freeform deformation. Finally the parameterization is represented by the coordinates of the lattice box points controlling the freefrom deformation (FFD), see Figure 1. One of the main advantages with the present approach is the ability to create smooth surfaces with a low number of lattice box points. In general freeform deformation is performed in two essential steps. At first all vertex points of the initial surface grid in cartesian space are mapped into an initial trivariate B-spline volume, Figure 2. In a second step the initial B-spline volume is replaced by a new B-spline volume with new knot points. All grid vertices are then remapped to cartesian space to yield a new surface. Consequently FFD can be deemed as an indirect method since the changes of the shape is at last determined by the changes of the control point lattices, Figure 3.

This approach made it necessary to split the mesh deformation modul into two parts. The first part has been demonstrated above and the second part deforms the grid points in the flow field with an advancing front algorithm, Ref. ${ }^{31}$ The advancing front algorithm is fast and robust even if large surface movements are required, as seen in Figure 3. 


\begin{tabular}{|c|c|c|c|c|c|}
\hline Design & Lattice & FFD & \multirow{2}{*}{$\begin{array}{l}\text { Surface Grid } \\
\text { Deformation }\end{array}$} & Advancing front algorithm & \multirow{2}{*}{$\begin{array}{l}\text { Volume Grid } \\
\text { Deformation }\end{array}$} \\
\hline Parameter & Box & & & & \\
\hline
\end{tabular}

Figure 1. Workflow for grid deformation.

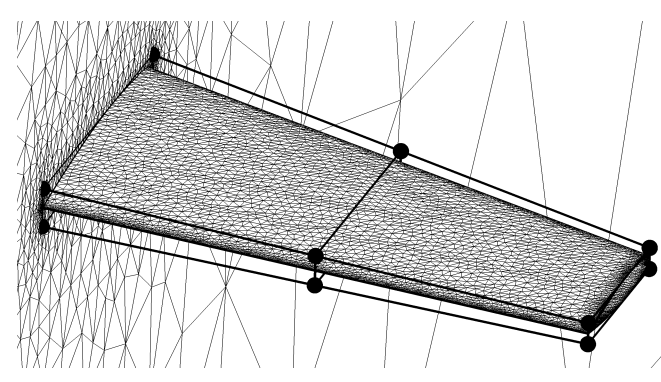

Figure 2. ONERA M6 wing with the initial lattice box

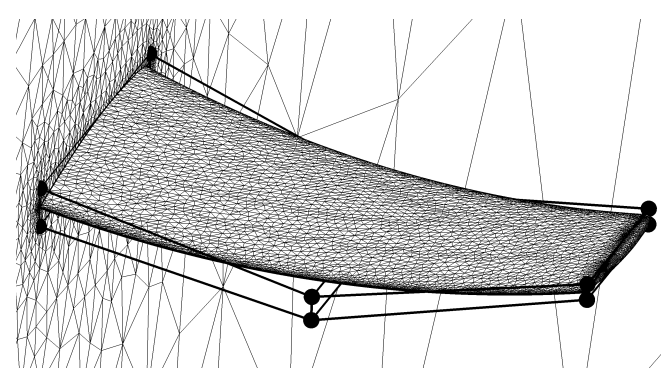

Figure 3. Deformed ONERA M6 wing with adjacent lattice box

\section{II..3. Validation of the adjoint sensitivities}

For validation purposes we test the described approaches with an ONERA M6 wing geometry, Ref. ${ }^{30}$ shown in Figure 2. The grid contains 108,396 points and 582,752 tetrahedra. The freestream Mach number is 0.84 and the angle of attack is 3.06 degree. The computation was done on a Linux Opteron computer with dual core processor with an semi-implicit LUSGS scheme and the flow and the adjoint simulation has been converged to $10^{-7}$ for the density residual and the first adjoint variable, either for the drag and lift cost function.

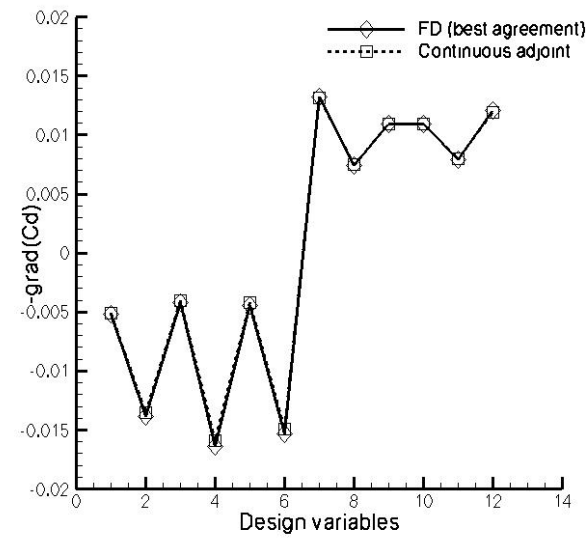

Figure 4. Comparision of sensitivities between finite differences and adjoint (drag)

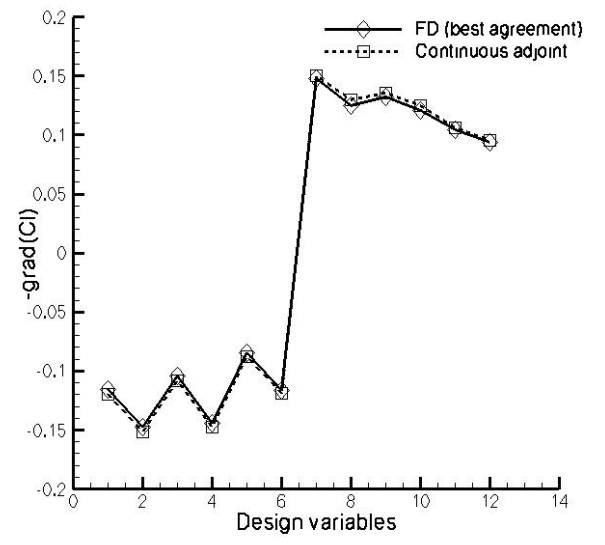

Figure 5. Comparision of sensitivities between finite differences and adjoint (lift)

The sensitivities were determined for 12 design variables according to the points of the lattice box. A finite difference method is used to compare the sensitivities obtained by the adjoint method. The comparison between the gradients, shown in Figure 4 and 5, demonstrates that the gradients are accurately represented by the adjoint method. The same work-flow can now be adopted for more complex geometries.

\section{II..4. Validation of the optimization process}

To show the efficiency of the Euler continuous adjoint method and freeform technique an optimization is performed with the ONERA M6 wing. The cost function was the drag while keeping the lift constant and 
will result in a maximization of the lift-to-drag ratio $L / D$. The lattice box contains 60 points of which a subset of 48 points are used as design parameters, Figure 6 . The lattice points at the root of the wing were kept fixed to prevent the wing becoming too thin. The other constraints are constant lift and pitching moment.

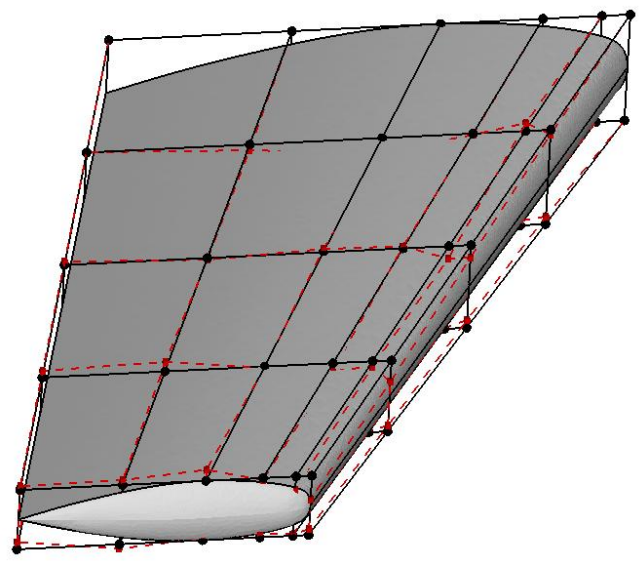

Figure 6. ONERA M6 with the initial and deformed lattice points defining the design variables

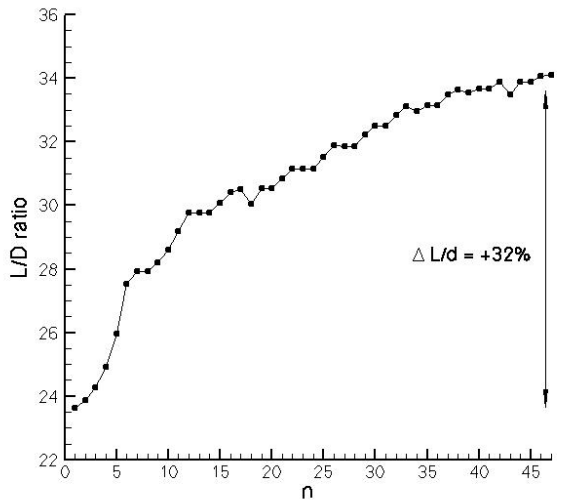

Figure 7. Lift-to-drag ratio during optimization of configuration

This case has been run again on the same Linux Opteron machine for 28 hours and the flow and associated adjoint solver has been converged to density residual and first adjoint variable to $10^{-8}$. The optimization required 10 sensitivity analysis. Results for the lift-to-drag ratio are shown in Figure 7 and the final value value is approximately $32 \%$ higher than its initial value. The Figure 8 and Figure 9 show the Mach number distribution on the surface from the initial and final geometry. As expected, the $\lambda$-shock vanished completely.

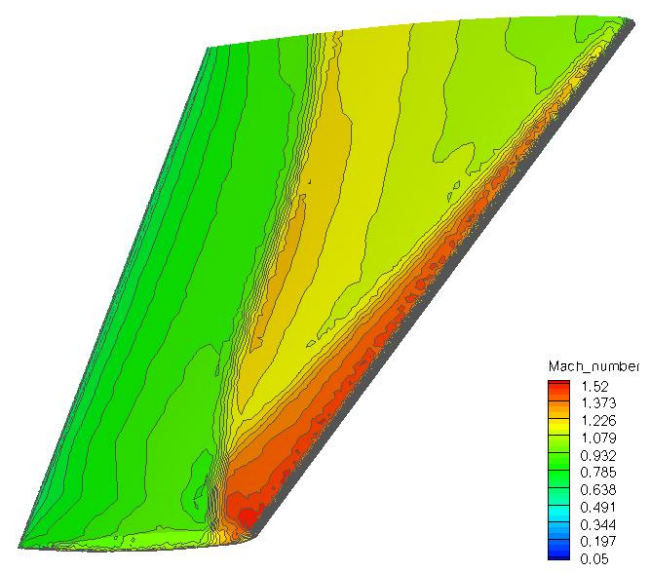

Figure 8. Mach number distribution over the surface for the initial grid

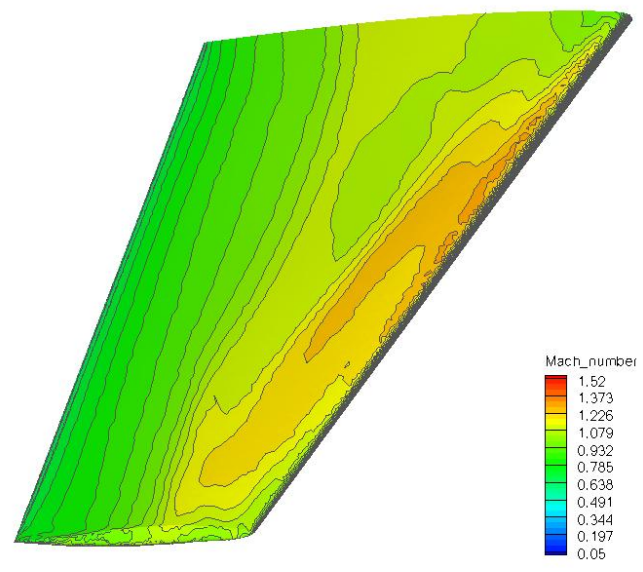

Figure 9. Mach number distribution over the surface for the optimized grid

This test-case gives a first idea of the validity of the adjoint implementation coupled with an appropriate surface parametrization and grid deformation strategy. The good convergence of the optimization process and the resulting design confirm the high accuracy of the gradients computed with the continuous adjoint method. 


\section{Optimization of a Large Flying Wing Aircraft}

The described optimization procedure is now applied to a blended wing body aircraft. The initial geometry was originally developed within the European Project VELA (Very Efficient Large Aircraft) and was provided by Strüber and Hepperle. ${ }^{29,32}$ The aircraft is designed as a future passenger transport aircraft with long-haul capabilities.

The design approach by Ref. ${ }^{32}$ was performed with the structured flow solver FLOWer ${ }^{18}$ and a classical gradient free, Nelder-Mead ${ }^{22}$ Simplex method. The optimization performed covered an optimization for finding the maximum L/D ratio considering a given constant lift. During the VELA project it was observed that the Simplex method is a robust optimization strategy. Therefore we used a gradient free method as a reference to compare the gradient based optimizations. Unfortunately, a direct comparison of the results obtained by Ref. ${ }^{32}$ was not possible due to the differences between structured and unstructured grids and the regeneration of the grid during each optimization cycle.

The following computations present optimizations for minimization of the drag with for prescribed lift. The flight conditions are a free-stream Mach number of 0.85 and fixed angle of attack at 1.8 degrees. The first approach involves a pure planform optimization, the second covers a planform and camberline approach and the last optimization was a pure shape optimization. A passenger compartment inside the main wing forms a constraint to prevent the final geometry becoming to thin or not practicable

The optimizations, based on a complete unstructured approach, include the methods stated in Part II and either a gradient free, Subplex ${ }^{34}$ based Nelder-Mead ${ }^{22}$ method and a modified method for feasible directions (MMFD) optimizer using an adjoint method. The initial geometry is depicted in Figure 11 and shows the planform of the initially used geometry. The lattice boxes for the freeform deformation were generated with DLR's parametric grid generator MegaCads, ${ }^{4}$ whereas the grid for the TAU computations was generated with the software package Centaur. ${ }^{5}$ The grid contains approximately 1 million points.

The main focus of the following comparisons lie on the results obtained for different design parameters, which have e.g. high (planform) and small (camberline) deformations. In this paper we neglect the evident changes in the structure and presumably in the weight of such a flying wing aircraft. The constraints for the global coefficients are set up with a very small range around the initial lift coefficient and the pitching moment.

Concerning the adjoint approach, here shortly referred as Adjoint, the sensitivity evaluation is almost independant of the design parameters. But as it is true for all gradient methods it can be trapped into a local minimum, while a Subplex is costly whenever the number of design parameters exceeds a certain amount but due to its approach is maybe able to find a global minimum. A comparison implies therefore a description of the optimization loop, the results from each particular optimization and the time needed to perform the optimizations.

\section{III.A. Flow chart of the optimization loop}

The optimization loop for the gradient algorithm is depicted in Figure 10 (left) together with the Subplex algorithm (right). Both charts start by building the freeform deformation boxes around the CAD-model with MegaCads and the Grid Generation of the initial unstructured grid. The single run of the deformation prepares the NURBS Parametrization for the freeform deformation, which is saved to disk. Subsequently the Deformation module and the Flow Solver are executed to deliver the first flow solution. Initially the deformation is zero. From now on both methods differ.

The Subplex method continues with the Analysis from the global coefficients derived from the flow computation and checks the prescribed constrains, e.g. the lift coefficient. If a constraint is violated during the minimization a Penalty Function adds a predefined value to the Objective Function for controlling indirectly the Optimizer. The Optimizer updates the Design Parameters until an optimum is reached. If not, the batch version of MegaCads, MegaBatch, deforms the lattice box according to the new parameter settings. Now the loop can be closed by performing the Deformation again. Alternatively, because the Subplex method is not bound to the same grid topology, the loop can be expanded to proceed further with the Grid Generation.

The gradient based loop decides, after obtaining the global coefficients from the Flow Solver, for the necessity of a Gradient evaluation. If necessary, an adjoint computation is performed. Additionally, when constraints are active, e.g. lift and/or pitching moment, the adjoint variables of these constraints have to be computed too. The Sensitivity computation, described in Part II..1 and II..2, finishes the gradient evaluation. The Optimizer may execute a quasi Newton iteration or a line search within a steepest decent or a SQP and 

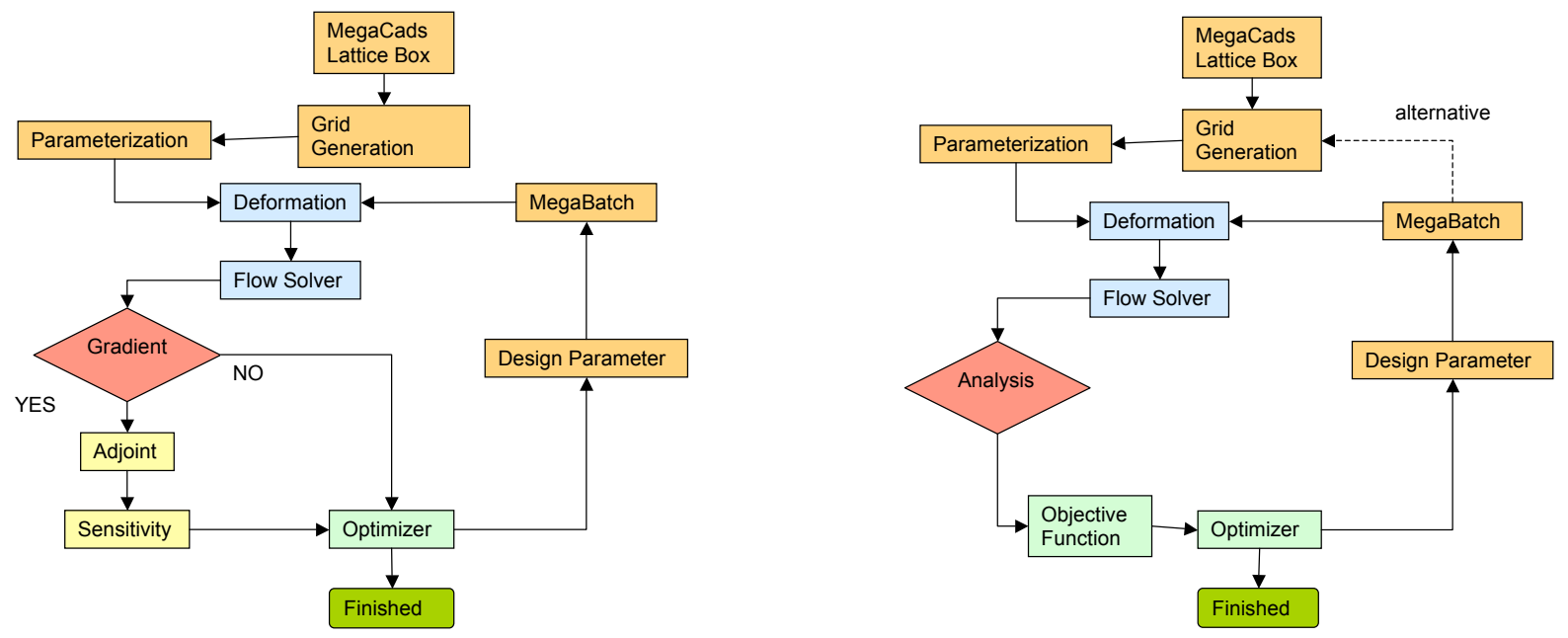

Figure 10. Flow chart for the adjoint (left) and Subplex (right) optimization loop.

updates the Design Parameters. Again, MegaBatch deforms the lattice box and which is directly delivered to the Deformation tool which closes the gradient based optimization loop.

\section{III.B. Optimization Part I covering only planform parameters}

The first optimization performed with the VELA configuration demonstrates the capability of each optimizer involving design parameters for changing the planform. Planform deformations can be performed efficiently with less parameters but as expected highly deformed grids may result. The design parameters for changing the planform, Figure 12, are the two sweep angles $\varphi_{1}$ and $\varphi_{2}$, two variations of the chord length in the middle and at the tip of the outboard wing and three twist angles again for the outboard wing. The maximum variation of the sweep angles are for $\varphi_{1} \pm 8^{\circ}$ (initial: $55^{\circ}$ ) and for $\varphi_{2} \pm 15^{\circ}$ (initial $35^{\circ}$ ). The chord length variations were limited to a multiplication factor of 1.2 to 0.5 and the twist of the outboard wing at section III to $\pm 2^{\circ}$, at section IV to $\pm 5^{\circ}$ and at section $\mathrm{V}$ to $\pm 10^{\circ}$. The lift should be held constant and the margins for the lift coefficient is set to a maximum of $1 \%$ loss of lift, see top Figure 14. The pitching moment increase is limited to $1 \%$, too. The lower part of Figure 11 shows the initial freeform lattice box which is used in combination with the design parameters to deform the surface.

Figure 13 presents the reduction of drag. The gradient method obtained 16.9 drag counts using 82 cycles and the Subplex obtained 17.1 drag counts using the maximum allowed cycles of 260. Both constraints where kept inside the possible margins.

The history of the design parameters can be seen in Figure 15 and 16. Both applications had the highest variations for the sweep angle $\varphi_{2}$ and both chord length scalings. Increasing the sweep for the outboard wing corresponds with the physical behavior of the flow for decreasing the strength of a shock on the wing. Another reason might be the kink between the center and outboard wing.

The gradients with respect to the design variables of the drag coefficient can be seen in Figure 17. The first gradient distribution at cycle 1 shows the highest gradients for the sweep angle $\varphi_{2}$ and the chord length variation at section IV. At later cycles the gradient distribution becomes more even and the cycle interval between the evaluation becomes smaller. Experience has shown that the interval between the first and second (Cycle 54) evaluation is somehow unusual because expectations reveal a much smaller cycle interval. The gradients for the twist angles remain small and approximatly constant the complete optimization and therefore has less influence as can be seen from the twist angle history in Figure 15. The changes of the last gradients (Cycle 60,72) indicate a settlement and the optimization will shortly finish. For comparison Figure 18 shows the Mach number distribution of the initial VELA configuration, exhibiting a the strong shock on the upper and lower side of the outboard wing.

The surface pressure distributions, Figure 19, for the outboard wing sections reveal for the Adjoint and Subplex almost identical results by diminishing the shock considerably for section IV and V. The center wing sections I and II are not discussed here because changes were to small to point out differences. The final 

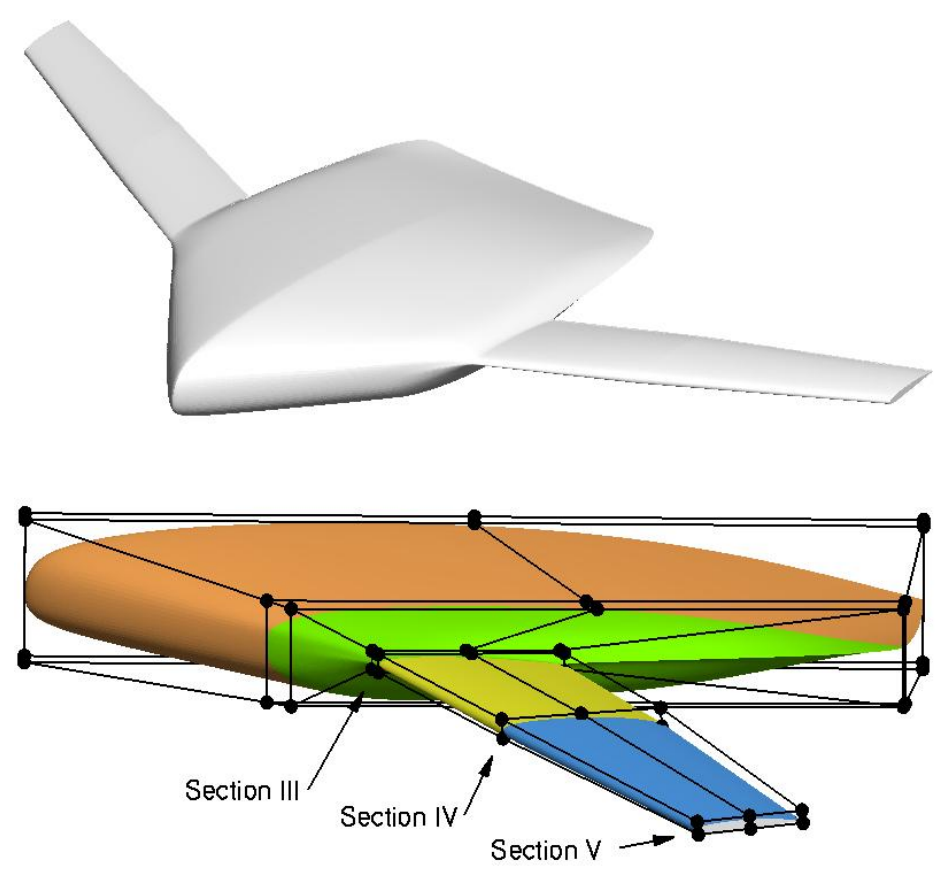

Figure 11. VELA configuration (above) and initial lattice box used for deformation.

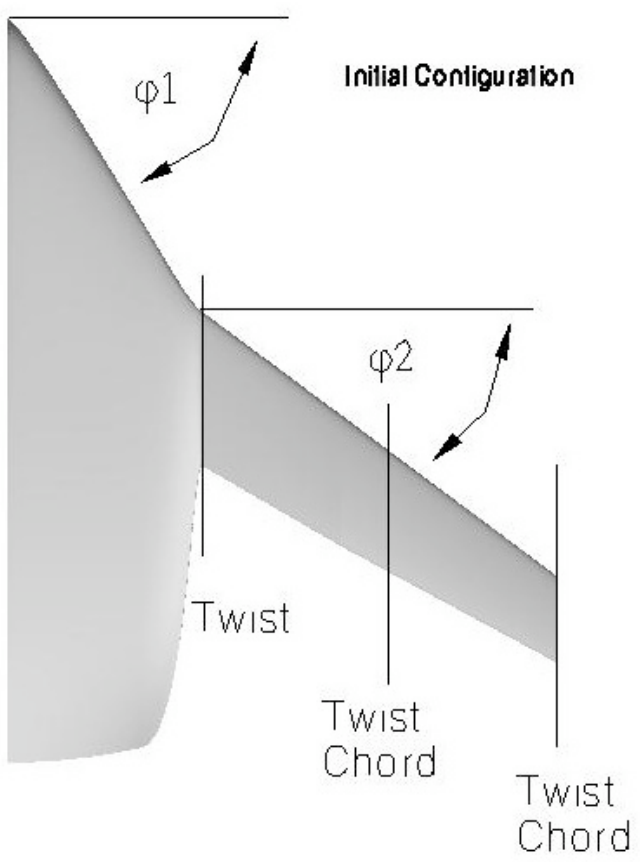

Figure 12. VELA configuration depicting the 7 design parameters.

comparison between the Adjoint and Subplex method is presented in Figure 20 for the surface Mach number distribution on the upper and lower side. To show the changes in the planform in conjunction to the sweep angles the initial planform is superposed as dotted contour on both pictures in Figure 20. As seen before from the pressure distribution, the shock on the upper side decreased at the outward side of the outboard wing but one can still see a strong displaced shock at the inner side for both approaches. On the lower side, near section III, the Subplex could reduce the shock strength more than the Adjoint.

Finally, both optimizations reached nearly the same drag count reduction by keeping the lift within the constrains. The resulting planform is very close to each other. It can be seen from the final planforms that the sweep angles are slightly different but both optimizations tried to line up leading edges of the inboard and outboard wings. For the gradient based method a successful optimization could be shown for design parameters only changing the planform which is comparable to the gradient-free approach. 

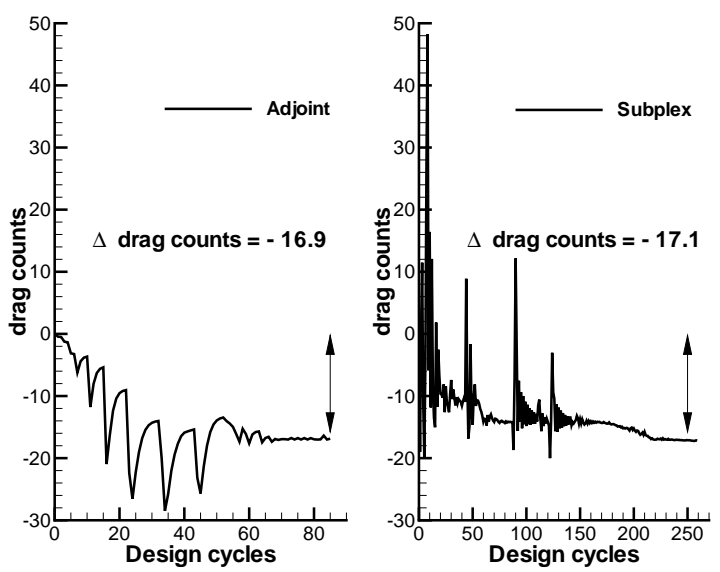

Figure 13. Reduced drag counts during a constrained optimization with 7 design parameters, Adjoint (left) and Subplex (right).
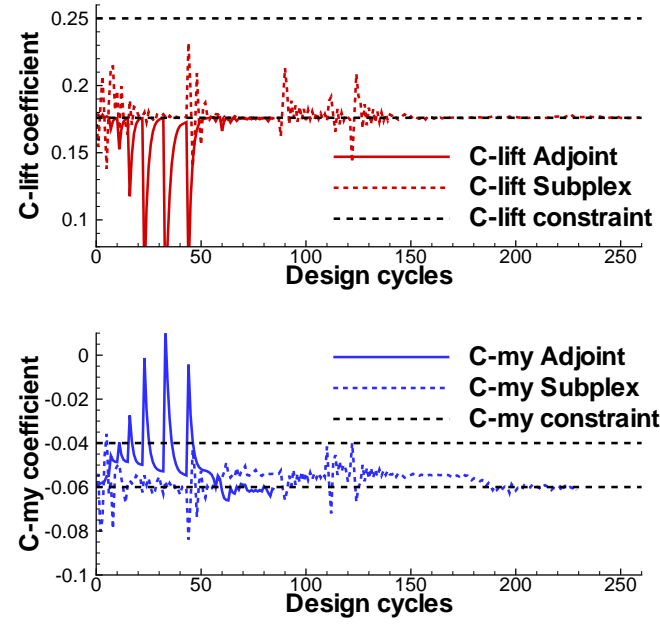

Figure 14. Lift (above) and Pitching moment (bottom) history during the optimization.
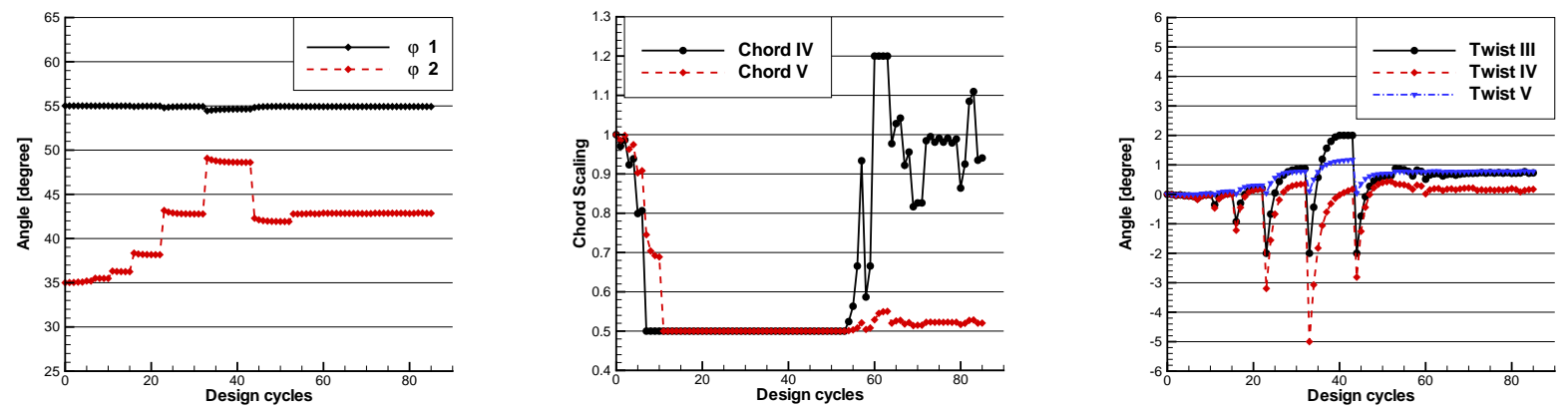

Figure 15. History of design parameters changing the Sweep angle, Chord scaling and the Twist angle from left to right for the Adjoint.
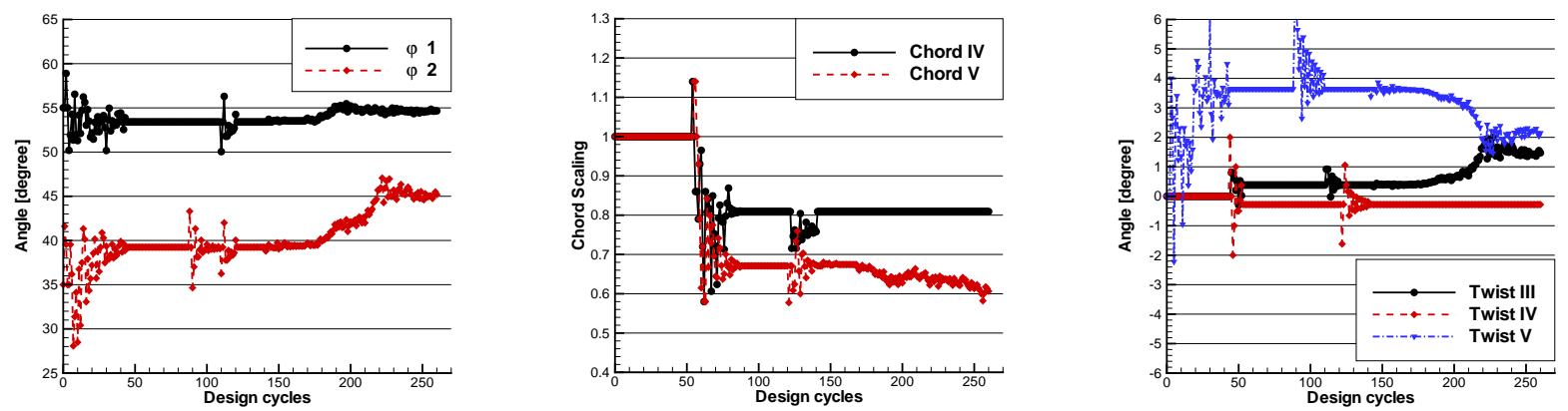

Figure 16. History of design parameters changing the Sweep angle, Chord scaling and the Twist angle from left to right for the Subplex. 


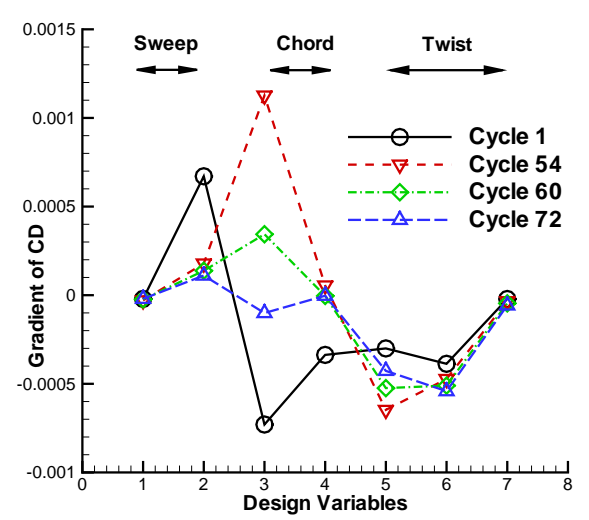

Figure 17. Gradients from the different optimization cycles with 7 design parameters.

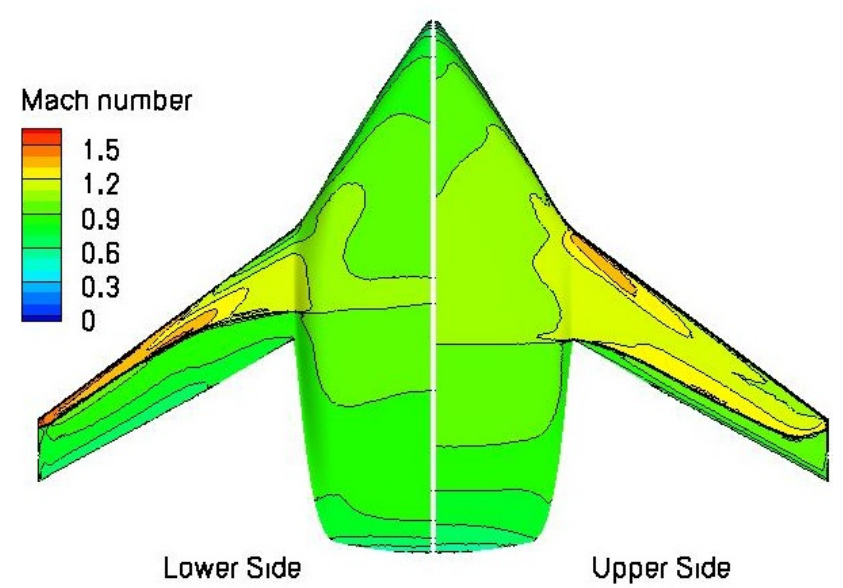

Figure 18. Initial Mach number distribution of the VELA configuration for the upper and lower side.
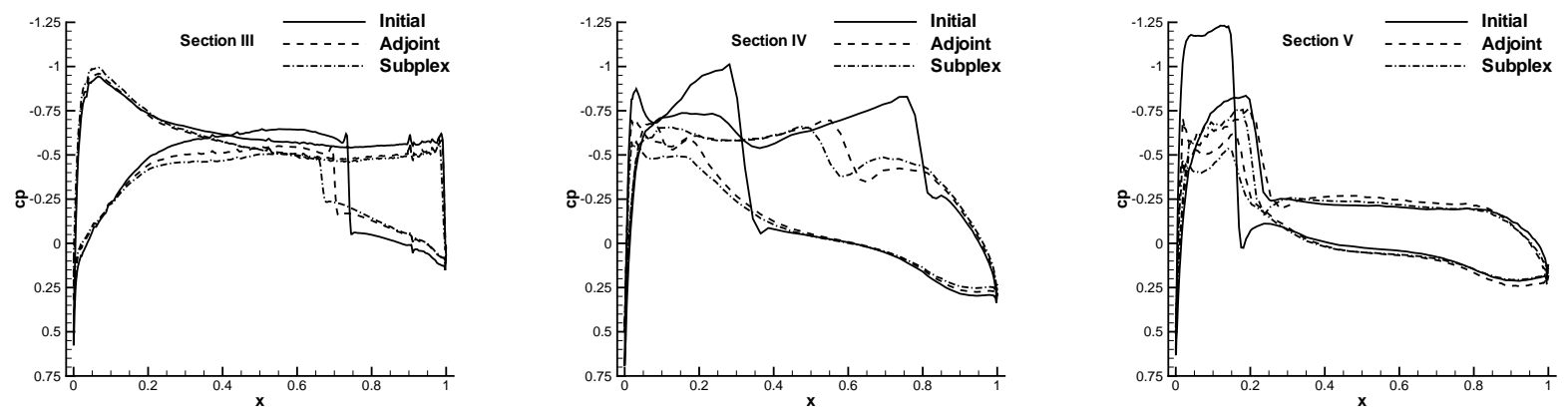

Figure 19. Comparison of the pressure distribution obtained from the initial configuration, Adjoint and Subplex on the parameterized chord length.
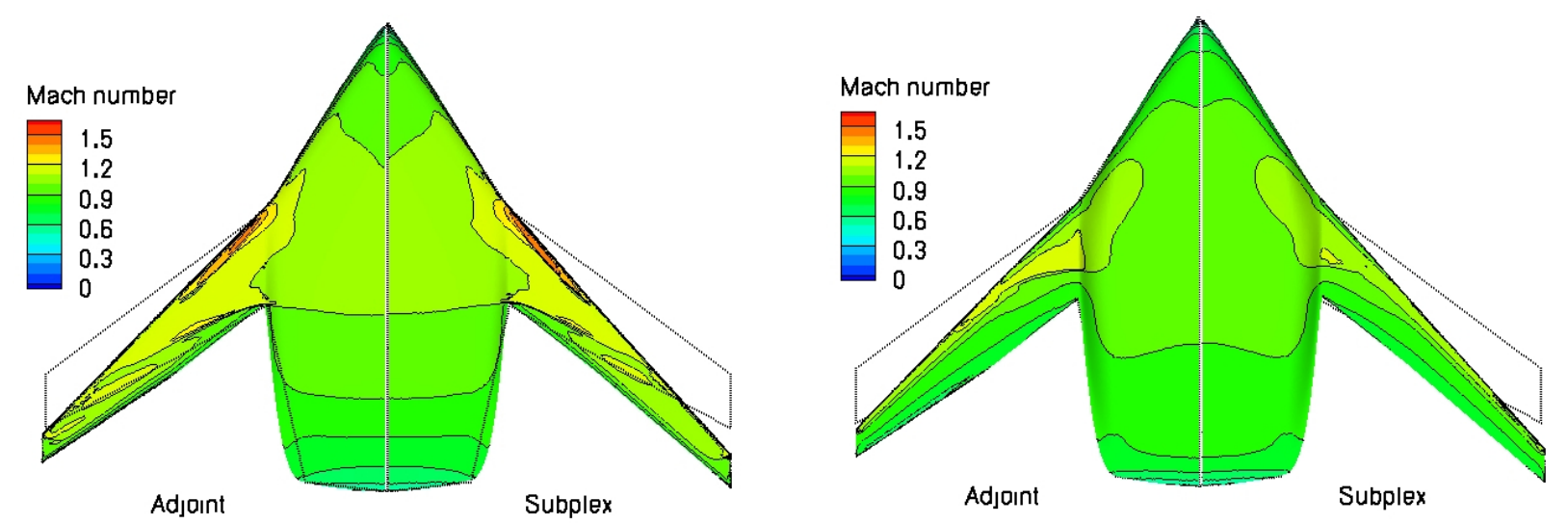

Figure 20. Comparison of the Mach number distribution obtained with the Adjoint (left) and Subplex (right), initial configuration is depicted with dots. 


\section{III.C. Optimization Part II covering planform and camberline design parameters}

This optimization is set up with design parameters changing the planform, two sweep angles $\varphi_{1}$ and $\varphi_{2}$, two scaling factors for the chord length at section IV and V and instead of the twist we introduced nine parameters changing the camberline of the outboard wing. The freeform deformation lattice boxes contain 3 design parameters in chord length direction at the top and at the bottom, Figure 21 and 22. In total this application will have 13 design parameters. The upper camberline parameters (red symbols) were allowed to move in z-direction (up and down) and the parameters, marked with black symbols, are shifted according to the upper parameters. The maximum movement was restricted to half the lattice box height.

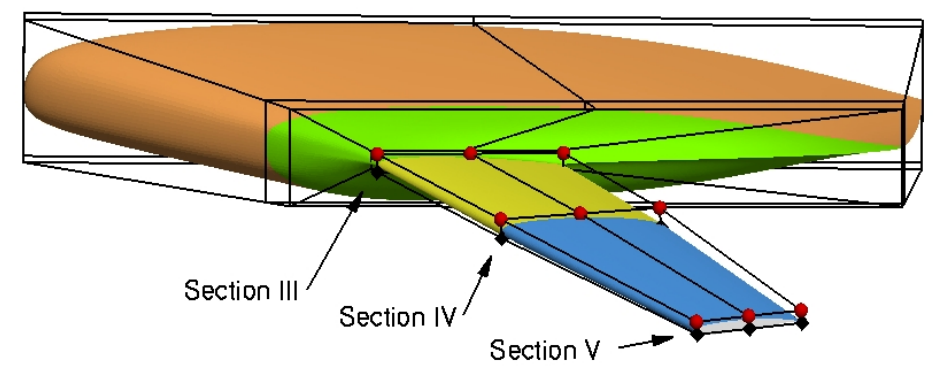

Figure 21. VELA configuration depicting the camberline design parameters (balls) acting at the Section III to $\mathrm{V}$ and accordingly shifted lattice points (diamonds).

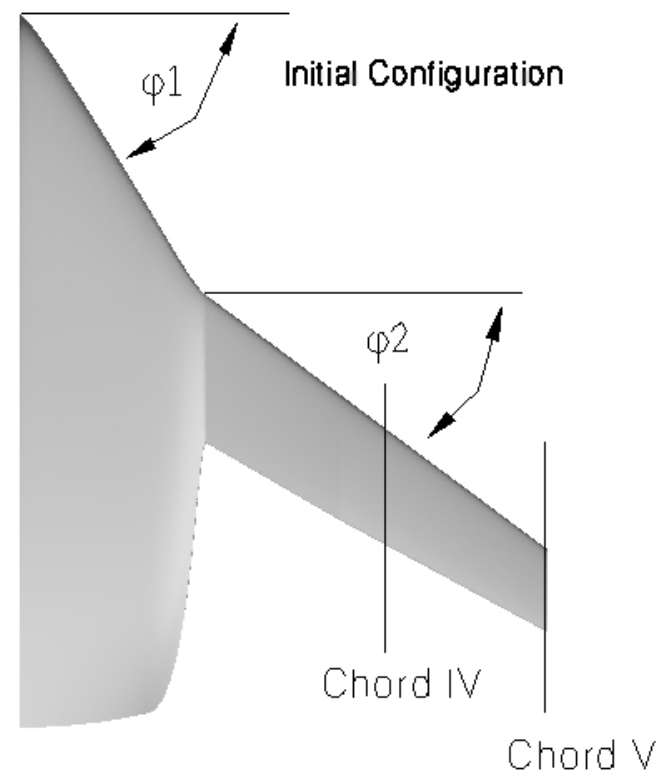

Figure 22. VELA configuration depicting the planform design parameters.

The different behavior of planform and camberline parameters is of great interest for the gradient based optimization due to their sensitivity evaluation. We have not introduced any scaling of the gradients to be able to compare both optimization results as they come with their original implementation.

Figure 23 presents the reduction of drag, 12 drag counts by the Adjoint and 15.7 drag counts by the Subplex method. The lift coefficient and the pitching moment were again inside the predefined margins, Figure 24.

The history of the design parameters can be seen in Figure 25 and 26. For better insight into the modified geometry, Figure 27 depicts the profiles and freeform deformation boxes of the initial and final planform and camberline in a parameterized perspective. In the following figures we can observe a completely different resulting geometry by comparing the planform and camberline. The behavior of the sweep angles for Adjoint method is remarkable. Both angles shift together with each other. The same behavior is evident with respect to the chord length scalings until the third gradient evaluation, comparing right Figure 25 and 28. The major influence from Adjoint is by shifting upwards and rotating the profiles clockwise. From the profiles in each section we experience a bending up to decrease the initial angle of incidence. The Subplex is mainly sweeping backwards and tries to bend up the profiles too, while only minor upwards shifting of the profiles could be detected.

Figure 28 shows the distribution of the gradients form the objective function (drag), during the gradient based optimization. The distribution is remarkable because the planform parameters are almost one order lower than the gradients from the parameters changing the camberline. But, as can be seen from the sweep angle and chord length history, Figure 25, and final profiles, Figure 30 (left), the changes of the planform parameters are not negligible. Contrary to the previous optimization is the cycle interval between the gradient evaluation which is spread almost equally approximately at each 10th cycle. The magnitude of the gradients are barely the same for the complete optimization.

Comparing the surface pressure coefficient $C_{p}$ for section III-V, Figure 29, with the surface Mach number 

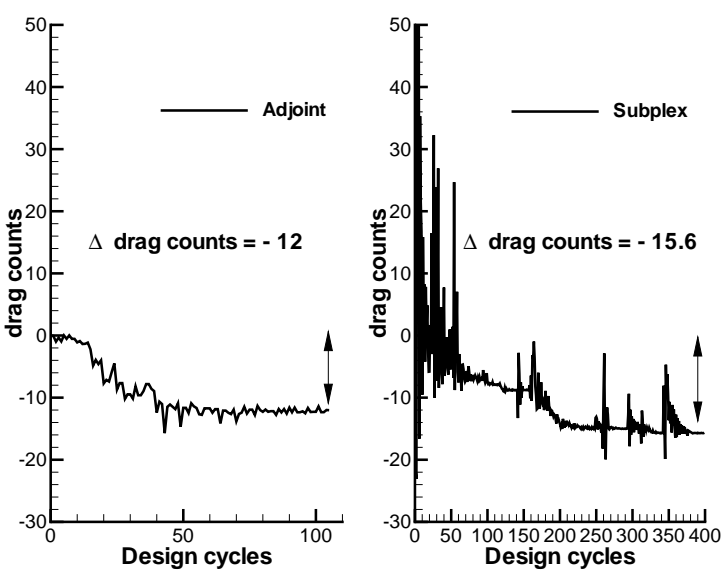

Figure 23. Reduced drag counts during a constrained optimization with 13 design parameters, Adjoint (left) and Subplex (right).
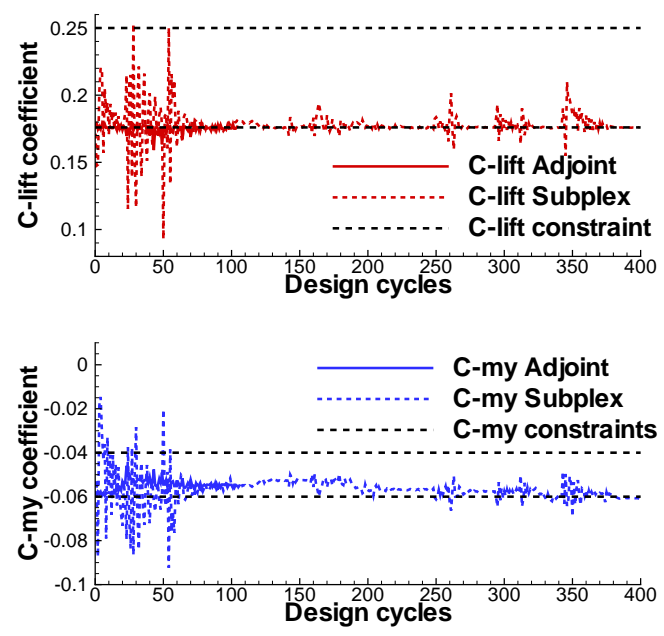

Figure 24. History of lift (above) and pitching moment (bottom) during the optimization.
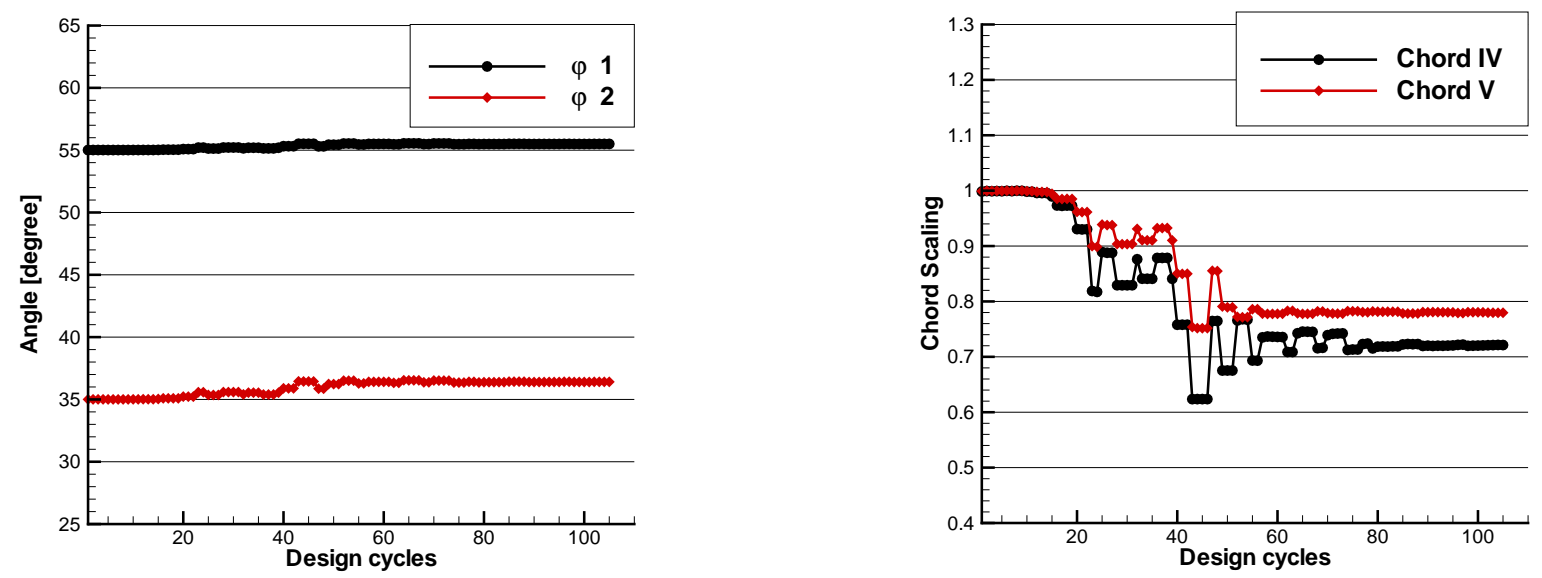

Figure 25. History of design parameter changing the sweep angle and the chord length for Adjoint.

distribution on the upper and lower side, Figure 30, it is obvious that the gradient method could not remove the shock seen at section IV on the upper side. At section III $C_{p}$ remained much the same as for the initial configuration but at section $\mathrm{V}$ both methods reached approximately the same improvements. On the upper side the Suplex could suppress the shock almost entirely whereas the Adjoint moved the shock backwards and reduced it slightly. The lower side shows almost the same flow pattern and a weak shock can be identified in the middle of the outboard wings root, well visible at the first Figure 29.

For these two optimizations the results are completely different regarding each design parameter. The obtained drag reduction was about $4 \mathrm{drag}$ counts better for the Subplex than for the Adjoint. Finding an optimum is even for the Subplex not as trivial as is seems, because the maximum allowed 400 cycles were reached. In case of the Adjoint applying a scaling to the design parameters for the planform will be the basis for further investigations. 

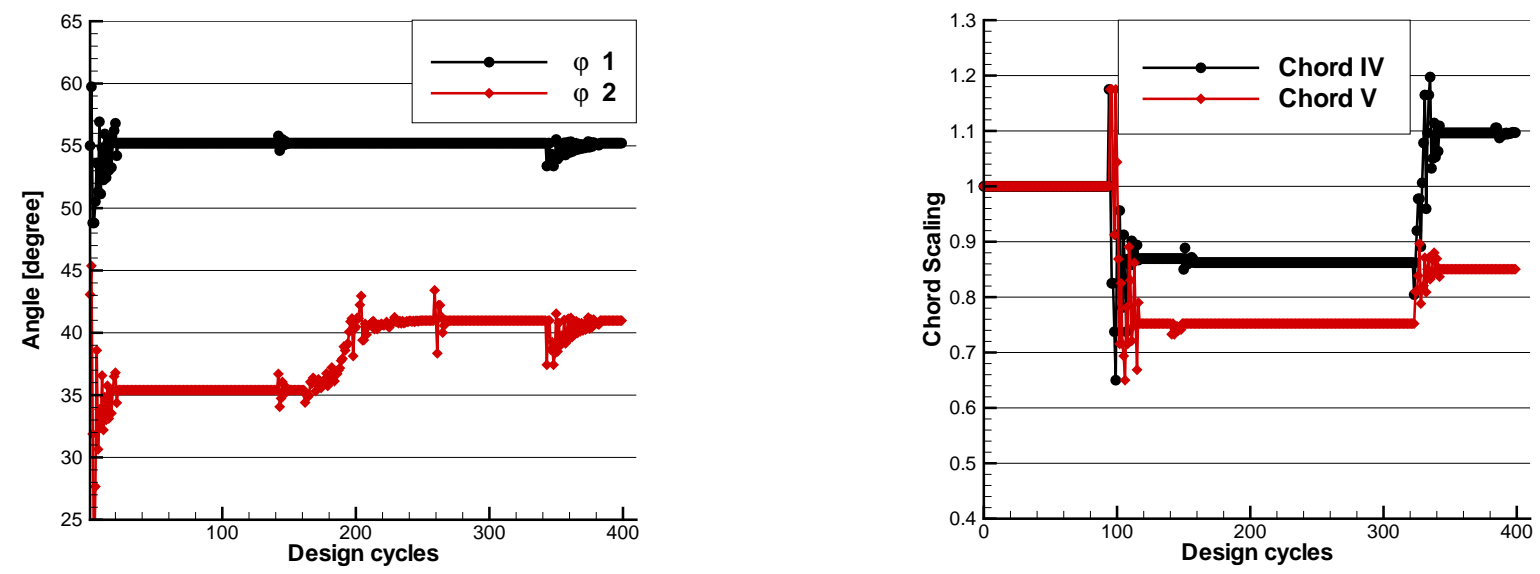

Figure 26. History of design parameter changing the sweep angle and the profile length for the Subplex.

Section III
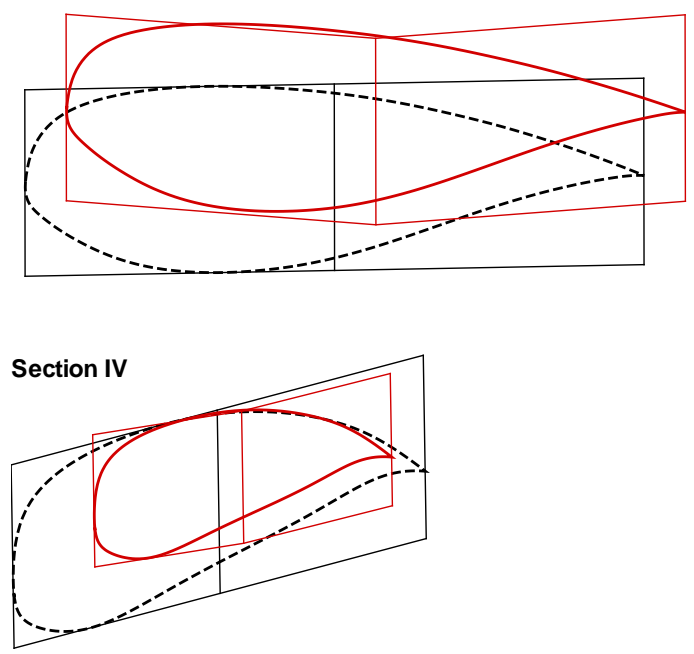

Section V

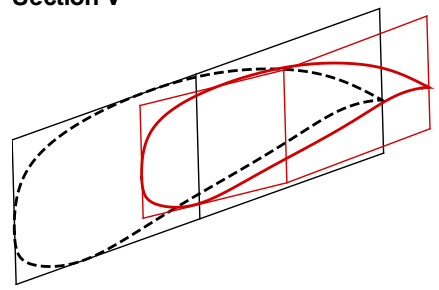

Section III
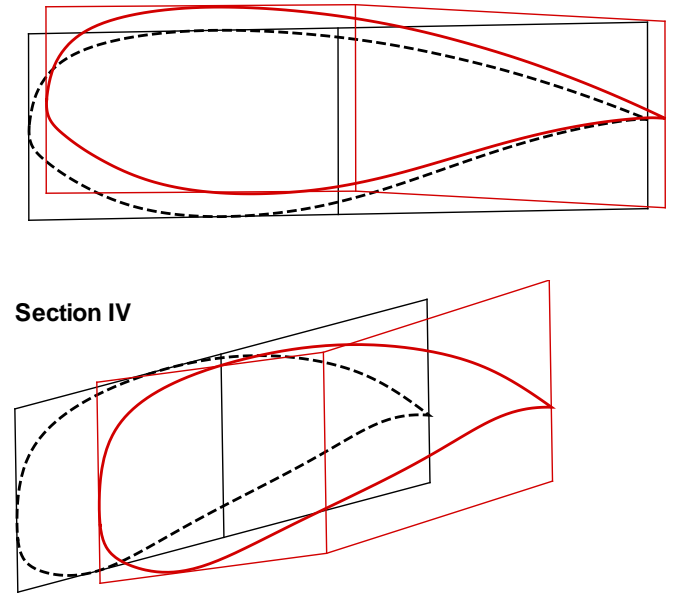

Section V

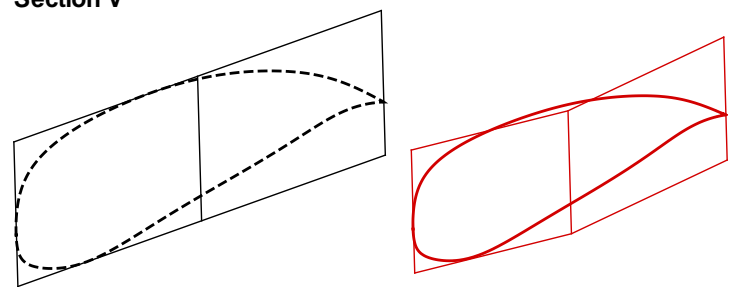

Figure 27. Parameterized outboard wing sections with the initial and deformed profile and each freeform lattice box for Adjoint (left) and Subplex (right). 


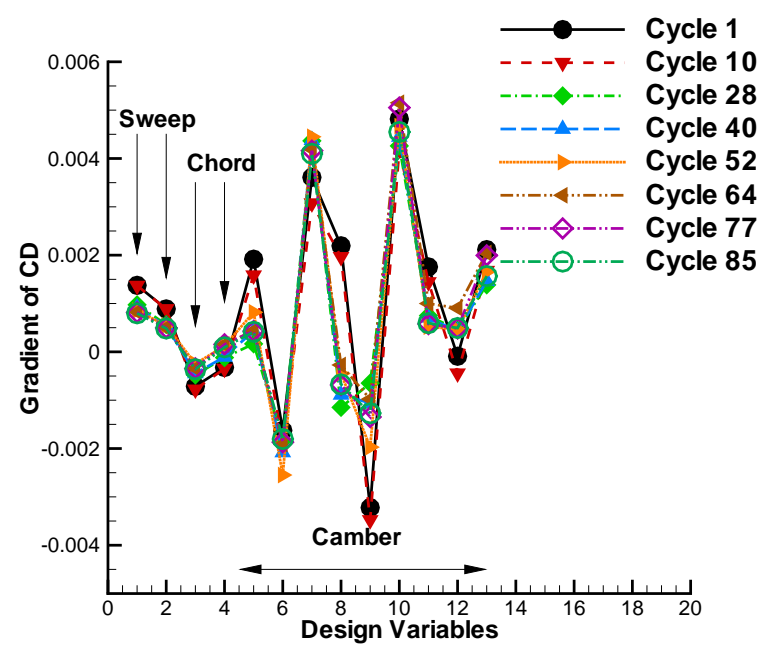

Figure 28. Gradients from the different optimization cycles with 13 design parameters.
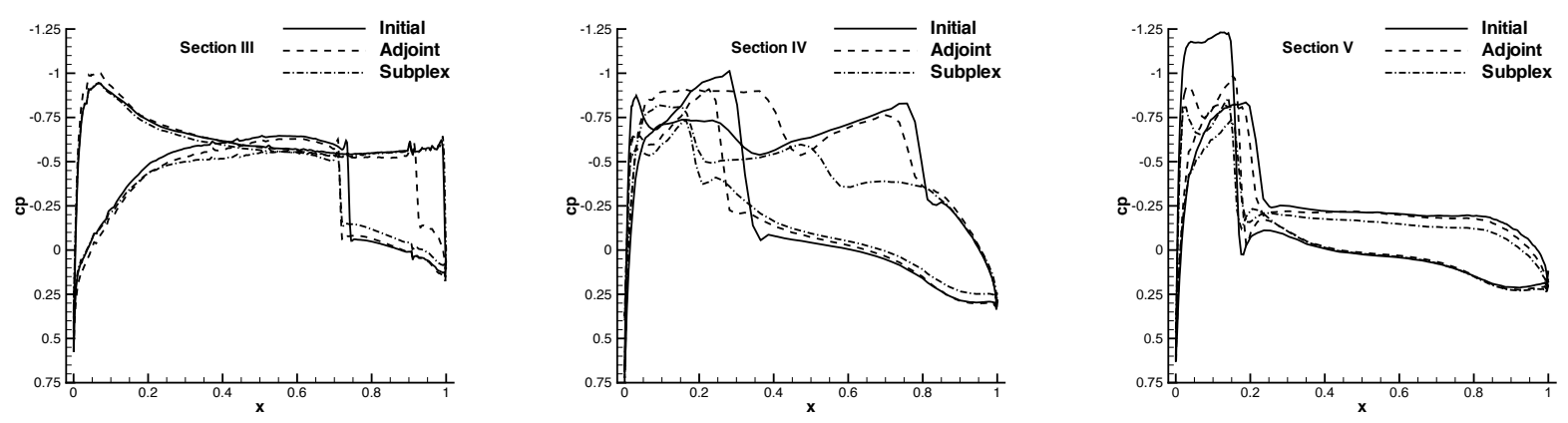

Figure 29. Comparison of the pressure distribution obtained from the initial configuration, Adjoint and Subplex on the parameterized chord length.
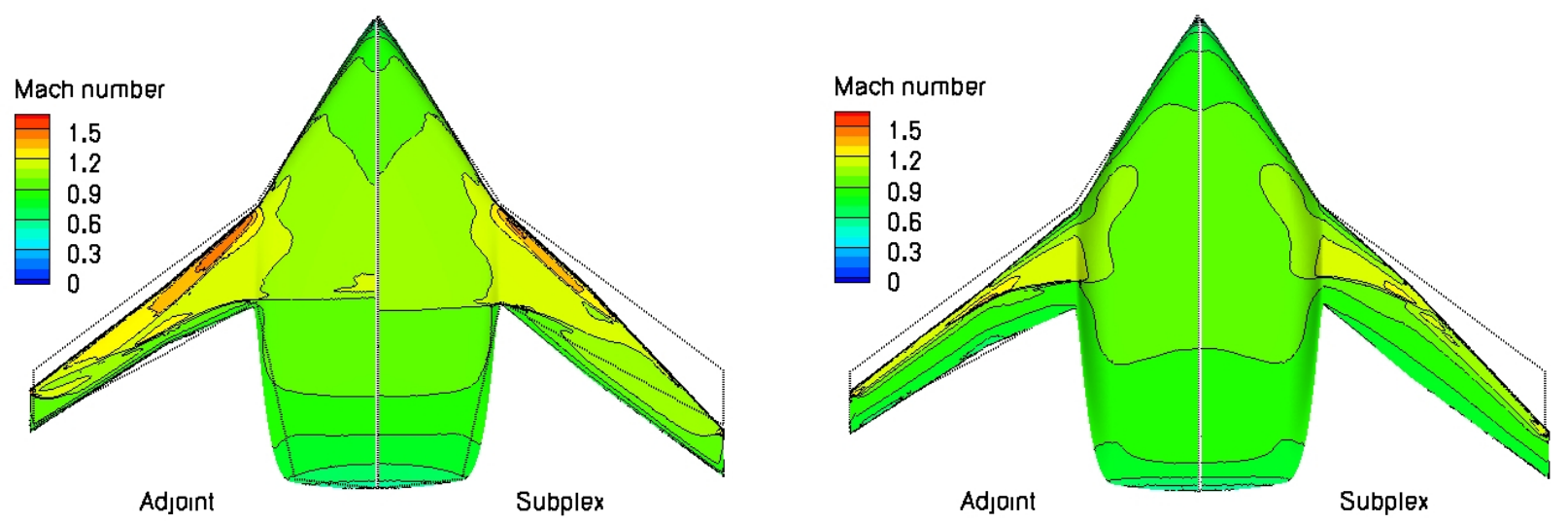

Figure 30. Comparison of the Mach number distribution obtained by application of the Adjoint (left) and Subplex (right), initial configuration is depicted with dots. 


\section{III.D. Optimisation Part III covers the full model for changing the shape}

This last application was made with the gradient based optimizer only and concerns a pure shape optimization. This optimization should point out the ability to introduce many design parameters for the adjoint method with little extra effort for the optimization procedure. The freeform deformation lattice boxes contain 5 design parameters in chord length at the top and at the bottom. Constraints for the design parameters of the center wing are defined by a passenger cabin, landing gear compartement and a cargo bay, Figure 32 . The outboard wing is constrained by keeping the center and rear freeform lattice points unchanged, Figure 31. In total this application has 30 design parameters, neglecting the fixed parameters as indicated by black diamonds in Figure 31. The design parameters were again allowed to move up and down and the maximum deflections were restricted to half the lattice box height. The margins for the design parameter changing the

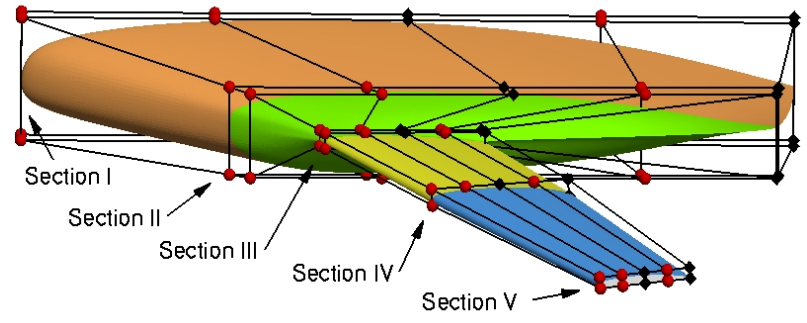

Figure 31. VELA configuration depicting the design parameters (balls) and fixed parameters (diamonds)

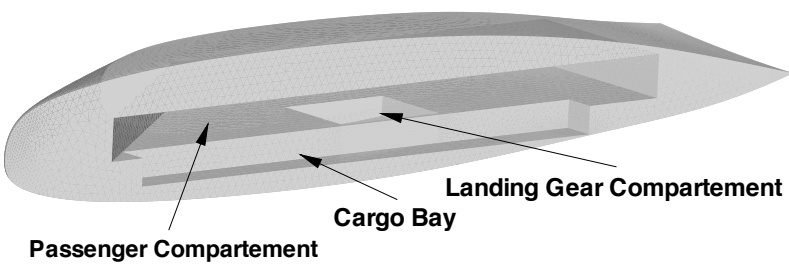

Figure 32. Grid with passenger and landing gear compartment in the center wing.

center wing had to be derived before starting the optimization. An extra tool providing the ability to find intersecting surfaces had been used to check intersections of the surface and the payload geometry. This tool was used to define the allowable parameter ranges. Most modern grid generators have this feature included. At the end of the optimization a final intersection check was done to check for a successful approach.

The optimization finished after 30 cycles. The obtained drag reduction was 10.5 drag counts, Figure 33. The constraints for the lift and the pitching moment are the same as for the previous optimizations and were satisfied for the lift coefficient while the pitching moment decreased by $6 \%$, Figure 34 . A change in the pitching moment was expected because the constraint was not handled as strict as the lift and no planform parameters are able to compensate for the loss of moment, whenever the flow pattern on the outbourd wing changes.

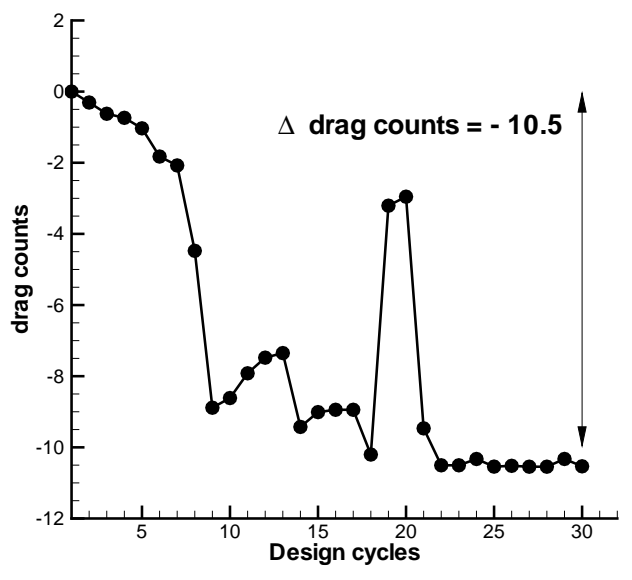

Figure 33. Reduced drag counts during a constrained optimization with 30 design parameters.
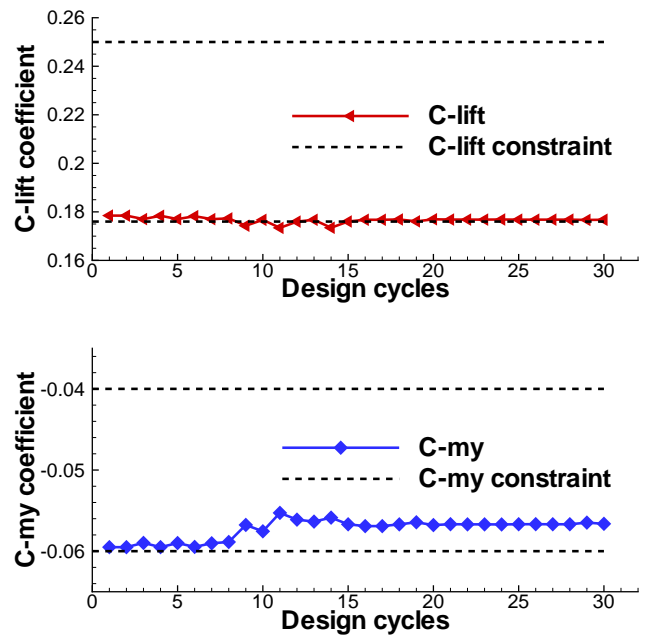

Figure 34. Lift (above) and Pitching moment (bottom) history during the optimization.

The parameterized wing sections I to V can be seen in Figure 35 and Figure 36. The first three sections 
in the center wing have small deformations as expected from the very restrictive compartments but tend to increase the nose radius while section IV and $\mathrm{V}$ are sharpening the nose and a move upwards. All representative sections show an S-type deformation, especially section IV and V to reduce the strong lower and upper shock at the outboard wing. Section $\mathrm{V}$ may turn into a rear loading profile. None of the lattice boxes reached the maximum deformation allowed.
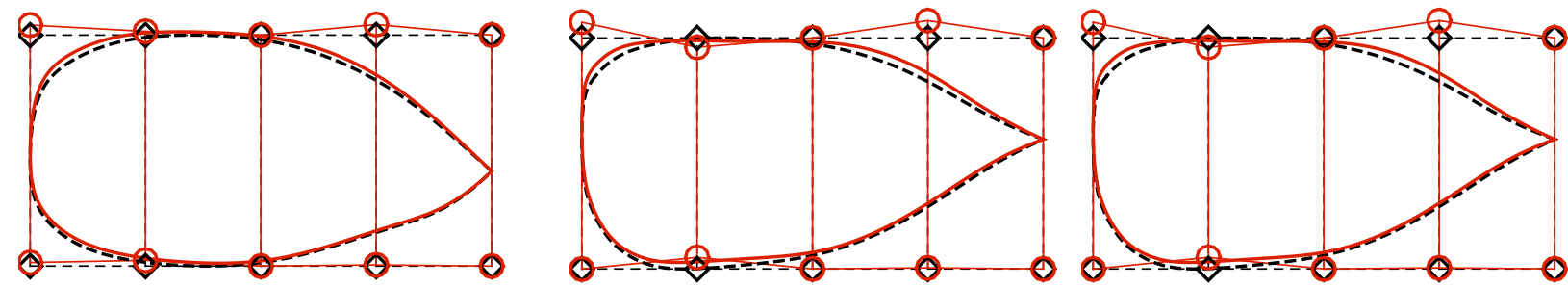

Figure 35. Parametrized lattice box and wing sections I,II and III for initial (dashed) and deformed (solid) grid.
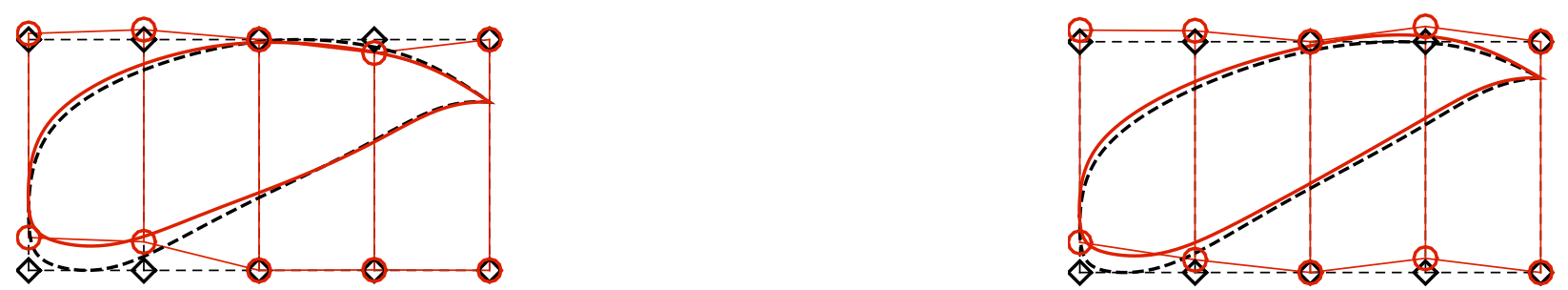

Figure 36. Parametrized lattice box and wing sections IV and V for initial (dashed) and deformed (solid) grid.

Figure 37 shows the distribution of the gradients of the objective function (drag) obtained during the optimization.

The surface pressure distributions $C_{p}$, Figure 38, 39 for section I - V in relation with the Mach number distribution, Figure 40, on the upper and lower side show a relevant reduction of the shocks apparent on both sides. The weak shocks on the upper side of the center wing have completely disappeared.

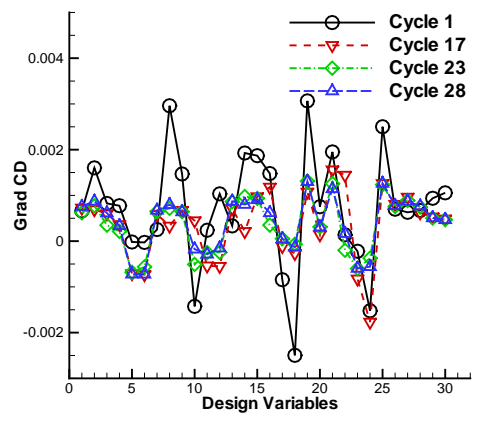

Figure 37. Gradients from the different optimization cycles with 30 design parameters.

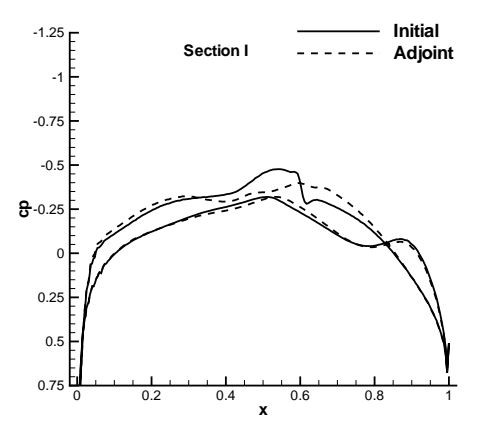

Figure 38. Comparison of the pressure distribution obtained from the initial configuration and Adjoint on the parameterized chord length.

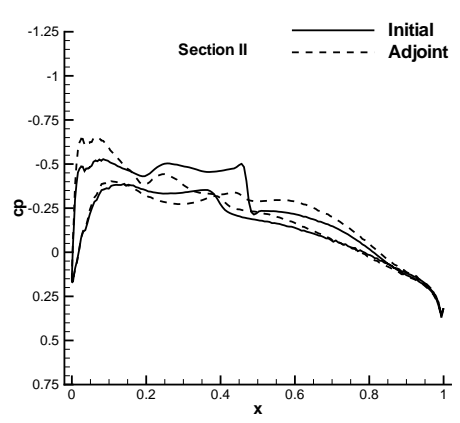

The most time consuming part for this application was the evaluation of the minima and maxima for the center wing design parameters to fulfill the compartment constraint which had to be done before the optimization. The realistic constraints, such like compartments in the center wing are restrictive in the sense that only small deformations are allowed. Nevertheless a drag count reduction of 10.5 was reached. 

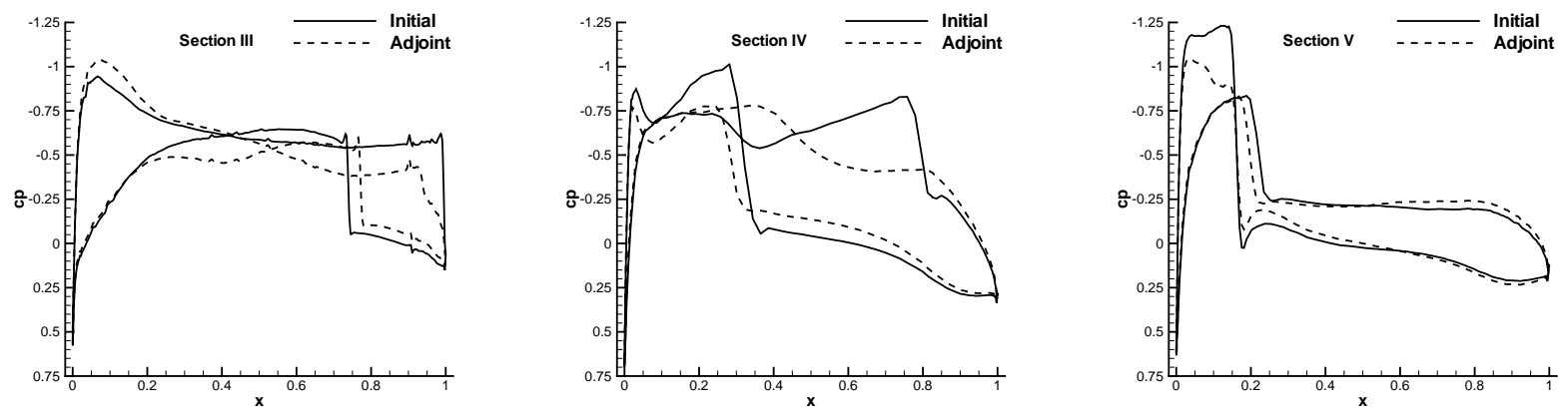

Figure 39. Comparison of the pressure distribution obtained from the initial configuration and Adjoint on the parameterized chord length.
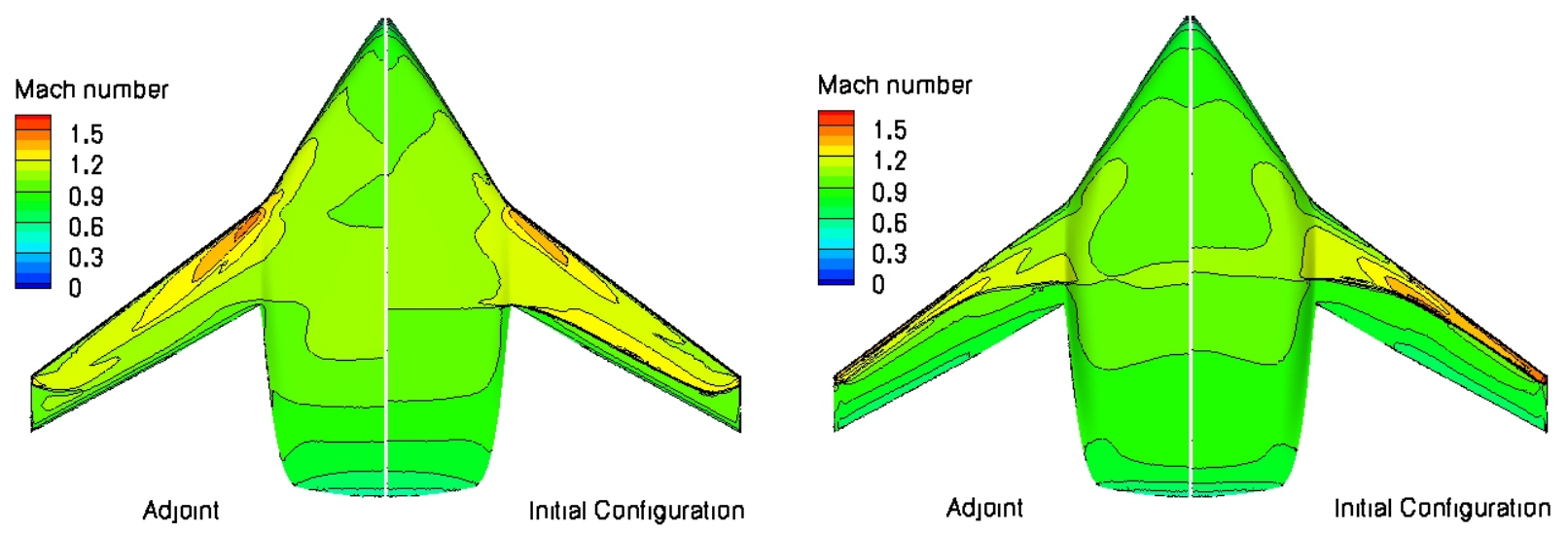

Figure 40. Comparison of initial (right) and final configuration (left) obtained by using Adjoint. 


\section{Computational Time for the different Optimization Cycles}

Time is a critical part for any optimization involving many design parameters. This is especially true in preliminary design. The optimizations were carried out on a Linux cluster using 4 processors in parallel for the flow and adjoint computations. Each flow analysis requires approximately 40 minutes while each adjoint analysis takes approximately 25 minutes for one evaluation of the cost function. The deformation of the volume grid was done in a sequential mode and took about 1 minute. All other procedures, like MegaBatch (see Figure 10 or the optimizer are negligible in terms of computational efforts.

Table 1 lists the time measured using seven respectively thirteen design parameters. Additionally, we have added the approximate time used for developing the scripts to run the deformation, flow solver, the objective function with the penalty for the Subplex, the gradient evaluation with Adjoint and their intensive testing for reliability. At first, it can be seen that the stepsize computation for best agreement, as needed for the determination of mesh sensitivities, takes a high percentage for the complete time of the gradient based method and has to be finished prior to any optimization. The stepsize computation covers 3 different steps, which is not sufficient but experience helped to shorten this part. The gradient evaluation took place 4 times with 7 design parameters and 6 times with 13 design parameters for three cost functions. The setup of the Subplex is more simple. The flow solver, adjoint solver and gradient evaluation sum up for the wall clock time, but the wall clock time is slightly higher due to extra time needed for the optimizer, MegaBatch and running the many scripts inside the loop and data transfer. In comparison, the wall clock time for the adjoint based optimization is much shorter then the Subplex approach.

\begin{tabular}{|c|l|c|c|c|c|}
\hline \multirow{2}{*}{ Procedure } & \multicolumn{2}{|c|}{7 design parameters } & \multicolumn{2}{c|}{13 design parameters } \\
\cline { 2 - 6 } & Adjoint $[\mathrm{h}]$ & Subplex $[\mathrm{h}]$ & Adjoint $[\mathrm{h}]$ & Subplex $[\mathrm{h}]$ \\
\hline \hline \multirow{3}{*}{ Prerequisit } & scripting, testing & 4 & 2 & 6 & 2 \\
\cline { 2 - 6 } & mesh step size & 36 & - & 64 & - \\
\hline \hline \multirow{3}{*}{ Optimization } & flow solver & 57 & 173 & 71 & 267 \\
\cline { 2 - 6 } & adjoint solver & 5 & - & 10 & - \\
\cline { 2 - 6 } & gradient evaluation & 0.8 & - & 3.2 & - \\
\cline { 2 - 6 } & WallClockTime & 64 & 175 & 86 & 269 \\
\hline \hline \multicolumn{2}{|c|}{ drag counts } & 16.9 & 17.1 & 13.4 & 15.6 \\
\hline
\end{tabular}

Table 1. Time consumption in hours for the optimization with 7 and 13 design parameters.

The time required for the optimization with 30 design parameters, Table 2, contains a large effort for finding the minima and maxima of the design variables of the center wing. The step size computation was done as mentioned above. The adjoint flow computation involved four gradient evaluations, again for three cost functions (drag, lift and pitching moment). Apart from the prerequisites, the optimization could be finished after 30 cycles by using only 26 hours.

\begin{tabular}{|l|c|c|}
\hline & Procedure & Adjoint $[\mathrm{h}]$ \\
\hline \hline \multirow{3}{*}{ Prerequisit } & scripting, testing & 16 \\
\cline { 2 - 3 } & mesh step size & 150 \\
\hline \hline \multirow{3}{*}{ Optimization } & flow solver & 20 \\
\cline { 2 - 3 } & adjoint solver & 5 \\
\cline { 2 - 3 } & gradient evaluation & 3.5 \\
\cline { 2 - 3 } & WallClockTime & 26 \\
\hline \hline \multicolumn{2}{|c|}{ drag counts } & 10.5 \\
\hline
\end{tabular}

Table 2. Time consumption in hours for the optimization with 30 design parameters.

The cost for the adjoint based optimization is driven by the computation to find the appropriate step size for the deformation of the grid to compute the mesh sensitivities with finite differences. A gradient 
evaluation with a finite difference approach with increasing design parameters is another part which strongly affects the computation times. This time consuming part can be eliminated by deriving the mesh sensitivities with an additional adjoint formulation for the mesh sensitivities. Approaches in that field have been done successfully by Nielson and Park $^{23}$ and Mavriplis. ${ }^{20}$

\section{Conclusion}

The capability of a gradient based method coupled with the continuous Euler adjoint formulation to perform a three-dimensional aerodynamic optimization has been shown in comparison with a gradient-free method. For a planform optimization only a small number of design variables is used but nevertheless the results of the adjoint method are available at each discrete grid point. In combination with the mesh sensitivities, evaluated by finite differences, the gradients could be determined in an accurate and straight forward way utilizing the freeform deformation. Apart from that, the freeform deformation deliveres a surface parameterization and deformation to generate the required smooth surface with a low number of design variables and this benefit can be exploited for more complex configurations. As shown for the gradient free approach, the freeform deformation can be used independently for any kind of optimization strategy.

The comparison of the optimization methods demonstrates that the gradient based method combined with the adjoint solver is a powerful alternative for preliminary design tasks. The request for initially developing an appropriate planform with few design parameters and finally optimizing in more detail with many design parameters and having fast solutions available can be met with the adjoint approach. Future work will now incorporate weight and balance and multipoint optimization to improve the off-design conditions.

Regardless of these advantages, further investigations will be arranged to pave the way to the usage of scaled gradients for applications with different sensitive parameters such as planform and camberline. Apart from the prerequisites of the adjoint technology, such as step size computation, the time needed for an optimization is within the time constraints of preliminary design.

In fact, an additional adjoint formulation for the mesh sensitivities, which is under development, will bring again a substantial improvement in the accuracy and a reduction of the time required for computing the gradients. In addition to this topic, future work will also concentrate on more sophisticated techniques for defining design parameters and adding more constraints to the design framework.

\section{References}

\footnotetext{
${ }^{1}$ Anderson, W. K.; Newman, J. C.; Whitfield, D. J.; Nielson, E. Sensitivity Analysis for the Navier-Stokes Equations on Unstructured meshes using Complex Variables. AIAA Journal, Vol. 39, No. 1, 2001, pp. 56-63

${ }^{2}$ Anderson, W. K. and Venkatakrishnan, V.: Aerodynamic Design Optimization on Unstructured Grids with a Continuous Formulation. AIAA Paper 97-0643, 1997

${ }^{3}$ Brezillon, J.; Gauger, N. R.: 2D and 3D aerodynamic shape optimization using the adjoint approach. Aerospace Science and Technology, 8, 8, pp. 715-727, 2004

${ }^{4}$ Brodersen, O.; Hepperle, H.; Ronzheimer, A.; Rossow, C.-C.; Schöning, B.: The Parametric Grid Generation System Mega Cads. 5th International Conference on Numerical Grid Generation in Computational Field Simulation, national Science Foundation, 1996

${ }^{5}$ Centaur Soft: http://www. Centaursoft.com

${ }^{6}$ Dwight, R.: Efficiency Improvements of RANS-Based Analysis and Optimization using Implicit and Adjoint Methods on Unstructured Grids. School of Mathematics, University of Manchester, 2006

${ }^{7}$ Dwight, R.; Brezillon, J.; Vollmer, D.B.: Efficient Algorithms for Solution of the Adjoint Compressible Navier-Stokes Equations with Applications Proceedings of the ONERA-DLR Aerospace Symposium (ODAS), Toulouse, 2006

${ }^{8}$ Galle, M.; Gerhold, T.; Evans, J.: Technical Documentation of the DLR TAU-Code. DLR-IB 233-97/A43 1997.

${ }^{9}$ Gauger, N. R., Brezillon, J.: Aerodynamic shape optimization using adjoint methods Journal of Aero. Soc. of India, Vol 54, No. 3, pp. 247-254, 2002

${ }^{10}$ Gerhold, T.; Galle, M.; Friedrich, O.; Evans, J.: Calculation of Complex Three-Dimensional Configurations employing the DLR TAU-Code. AIAA-97-0167, 1997.

${ }^{11}$ Giles, M.; Pierce, N.: An introduction to the adjoint approach to design. Proceedings of ERCOFTAC Workshop on Adjoint Methods, 1999

${ }^{12}$ Hager, J. O.; Eyi, S.; Lee, K.D.: Two-Point Transonic Airfoil Design Using Optimization for Improved Off-Design Performance. Journal of Aircraft, Vol. 31, 1994, pp. 1143-1147

${ }^{13}$ Hicks, R.M.; Henne, P.A.: Wing Design by Numerical Optimization. Journal of Aircraft, Vol. 15, 1978, pp. 407-412

${ }^{14}$ Jameson, A: Aerodynamic Shape Optimization Using the Adjoint Method. Lectures at the Von Karman Instiute, Brussels, 2003

${ }^{15}$ Jameson, A: Aerodynamic design via control theory. Journal of Scientific Computing 3 (3) (1988), pp. 233-260
} 
${ }^{16}$ Leoviriyakit, K.;Jameson, A.: Multi-Point Wing Planform Optimization via Control Theory. 43rd Aerospace Sciences Meeting and Exhibit, January 10-13, 2005, Reno, NV

${ }^{17}$ Kim, S.; Alonso, J.J.; Jameson, A.: Two-Dimensional High-Lift Aerodynamic Optimization Using Continuous Adjoint Methods. AIAA 2000-4741

${ }^{18}$ Kroll, N.; Radespiel, R.; Rossow, C.-C.: Structured Grid Solvers / Accurate and Efficient Flow Solvers for 3D Applications on structured meshes. AGARD Report 807, Special Course on parallel Computing in CFD, 1995

${ }^{19}$ Mavriplis, D. J.: Formulation and Multigrid Solution of the Discrete Adjoint for Optimixation Problems on Unstructured Meshes. AIAA-06, Reno, NV, vol. 44, pp.42-50

${ }^{20}$ Mavriplis, D. J.: A discrete Adjoint-Based Approach for Optimization Problems on Three-Dimensional Unstructured Meshes. AIAA-2006-50, 44th AIAA Aerospace Sciences Meeting and Exhibit, 9-12 January 2006, Reno, NV

${ }^{21}$ Monge, F. and Palacios, F.: Multipoint Airfoil Optimization Using Control Theory. Fourth European Congress on Computational Methods in Applied Sciences and Engineering, Jyväskkylä, 2004

${ }^{22}$ Nelder, J. A.; Mead, R.: A Simplex Method for Function Minimization. Comput. J. 7, pp. 308-313, 1965

${ }^{23}$ Nielson, E.; Park, M. A.: Using An Adjoint Approach to Eliminate Mesh Sensitivities in Computational Design. AIAA2005-0491, 2005

${ }^{24}$ Nielson, E.; Anderson, W. K.: Aerodynamic Design Optimization on Unstructured Meshes Using the Navier-Stokes Equations. AIAA-98-4809, 1998

${ }^{25}$ Ronzheimer, A.: Post-Parametrisation of CAD-Geometries Using Freeform Deformation and Grid generation Techniques. Notes on Numerical Fluid Mechanics and multidisciplinary Design, Vol. 87, pp. 382-389

${ }^{26}$ Ronzheimer, A.: Shape Parameterization in Multidisciplinary Design Optimization Based on Freeform Deformation. Evolutionary and Deterministic Methods for Design, Eurogen 2005, Munich

${ }^{27}$ Samareh, J.A.: Novel Shape Parameterization Approach. NASA TM-1999-209116, May 1999

${ }^{28}$ Samareh, J.A.: Aerodynamic Shape Optimization Based on Free-Form Deformation. AIAA 2004-4630, 2004

${ }^{29}$ Mialon, B.; Hepperle, M.: Flying Wing Aerodynamics Studies at ONERA and DLR. CEAS/KATnet Conference on Key Aerodynamic Technologies, 20-23 June 2005, Bremen

${ }^{30}$ Schmitt, V. and Charpin, F.: Pressure Distributions on the ONERA M6 Wing at Transsonic Mach Numbers Experimental Database for Computer Program Assessment AGARD-AR-138, May 1979, pp. B1-1-B1-44

${ }^{31}$ Schwamborn, D.; Gerhold, T.; Heinrich R.: The DLR Tau-code: recent applications in research and industry. Proceeding of ECCOMAS CFD 2006, Egmond aan Zee, Netherlands, 5.-8. September 2006.

${ }^{32}$ Strüber, H.; Hepperle, M.: Optimization of Flying Wing Transport Aircraft Configurations in the VELA Project. DLR-IB 124-2005/905

${ }^{33}$ Newman, J. C.; Taylor, A. C.: Three-Dimensional Aerodynamic Shape Sensitivity Analysis and Desing Optimization Using the Euler Equations on Unstructured Grids. AIAA 96-2464, 1996

${ }^{34}$ Wild, J.: Numerische Optimierung von zweidimensionalen Hochauftriebskonfiguration durch Lösung der Navier-Stokes Gleichungen DLR-FB 2001-11, ISSN 1434-8454

${ }^{35}$ Wu, H.-Y; Yang, S.; Liu, F.; Tsai, H.-M.: Comparison of Three Geometric Representations of Airfoils for Aerodynamic Optimization. AIAA 2003-4095, 2003 